\title{
STOCHASTIC VOLATILITY AND DEPENDENCY IN ENERGY MARKETS - MULTI-FACTOR MODELLING
}

\author{
FRED ESPEN BENTH
}

\begin{abstract}
We give a short introduction to energy markets, describing how they function and what products are traded. Next we survey some of the popular models that have been proposed in the literature. We extend the analysis of one of these models to include for stochastic volatility effects. In particular, we analyse a mean reverting stochastic spot price dynamics with a stochastic mean level modelled as an Ornstein-Uhlenbeck process. We include in this dynamics a stochastic volatility model of the Barndorff-Nielsen and Shephard type. Some properties of the dynamics are derived and discussed in relation to energy markets. Moreover, we derive a semi-analytical expression for the forward price based on such a spot dynamics. In the last part of these lecture notes we consider a cross-commodity spot price model including jumps. A Margrabe formula for options on the spread is derived, along with an analysis of the dependency risk under an Esscher measure transform. An empirical example demonstrates that the Esscher transform may increase the tail dependency in the bivariate jump part of the spot model.
\end{abstract}

\section{An introduction to ENERGy MARKETS}

There exist many markets for trade in power and related products. In Europe, Australia and the US, the markets for selling and purchasing electricity have been liberalized the last decades. For example, one has the NordPool market covering generation and distribution of power in the Nordic countries, and the German power exchange EEX. In the US, there are several markets, as well as new markets emerging in Eastern Europe and Asia.

Typical fuels for power generation are oil, gas and coal. Oil in different qualities has been traded for a long time at different exchanges, including for example NYMEX and ICE in London. In recent years, gas and coal have been traded at the ICE and EEX markets, opening for more competitive prices than in the more traditional bilateral markets. Usually, when talking of energy markets, one is thinking of the markets for power, gas, coal and oil.

With the recent decade's attempt to regulate climate gas pollution, a market for emission allowances have emerged. In Europe, one can for example trade allowances for the emission of carbon dioxide at the European Climate Exchange and EEX. A gas or coal fired power plant must match its emission of carbon dioxide over a year with allowances, which can be bought at the exchange. This introduces an additional variable cost to the production.

Most of the energy markets, including the emission markets, offer platforms for trading in futures and forward contracts as well as call and put options on these. This creates opportunities for the market participants to manage their risk exposure towards fuel costs and power prices. However, a major risk factor in the energy markets is weather. For example, in the Nordic region, the demand for power is very dependent on the temperature. Cold winter temperature leads to an increase in demand due to household heating. In the US, one has the similar effect of warm summer temperature, where the demand for power goes up due to air-conditioning cooling. On the other hand, rainfall fills up reservoirs for hydro power production, while wind gives rise to wind power generation. In many markets, both hydro and wind generation of electricity are major sources of power.

To manage weather risk, Chicago Mercantile Exchange (CME) organizes trading in temperature futures contracts written on weather indices measured in various cities world-wide. The typical

Date: February 25, 2013.

Financial support from the project "Energy Markets: Modeling, Optimization and Simulation" (EMMOS), funded by the Norwegian Research Council under grant 205328/v30, is greatly acknowledged. An anonymous referee is thanked for careful reading of the paper and the several suggestions which improved the presentation. 
temperature indices are the cumulative amount of heating or cooling degree days. In addition to temperature futures, one can trade in options on these futures at the CME. In 2007, the US Futures Exchange announced that they would start a market for wind index futures contracts written on 7 regions in the US where there are wind farms in 2008, however, the exchange closed down before this market came to be.

The power markets are interconnected through transmission lines. For example, one can send power produced in the Nordic countries to the German market, and vice versa, through air or sea-bed cables. This creates a dependency between the EEX and NordPool power prices, since a big price difference can be exploited by the producers and retailers in these two markets. Such opportunities are of course limited by the capacity in the transmission lines. However, an interesting effect of these inter-market dependencies were observed in the fall of 2007 . Power prices at NordPool were higher than normal, attributed to the fact that from January 2008 carbon emission fees were to be introduced in the European Union, and power prices would increase in the German market. In the NordPool market, power producers could hold back production by storing water in the reservoirs and wait for the expected higher prices. This led to less supply in the autumn, and hence higher prices. The power spot prices in Germany, on the other hand, were not influenced before the emission fees became effective in January. The autumn prices for clean hydro power included emission fees in the Nordic market before these fees were introduced, while "dirty" power in the EEX area remained unaffected. The EEX is largely supplied by nuclear, coal and gas, and very little hydro power relative to the Nordic market. In fact, the NordPool and the emission market got connected via the transmission lines to the EEX market, although hydro power production does not emit carbon dioxide. We refer to Benth and Meyer-Brandis [11] for a discussion and mathematical modeling of this situation.

Transmission line capacity may also create price differences within a power market due to congestion. In the NordPool market, area prices are settled for each hour throughout the day to balance out the loss by transporting electricity through the network. For example, Norway may have up to 5 different prices for a given hour due to congestions between the different areas. Of course, the different area prices are highly dependent, but there exist also periods of high price differences even in neighboring areas.

The gas markets are also connected, via a network of pipelines for distribution of gas from the hubs to the various regions of Europe. LNG, liquefied natural gas, creates possibilities to transport gas from one continent to another by large tankers. Hence, producers and retailers have the opportunity to play on price differences, and ship the gas to the best markets. There is a market for freight called IMAREX, providing access to risk management tools for transportation. As coal is shipped from mines in Australia and Asia to continental Europe, the freight market will also play an important role here.

As we have already mentioned, weather impacts both demand and production of power, and therefore the power prices. A typical feature of electricity markets is the spiky behaviour of the prices. Occasionally, prices may rise by several hundreds of percent, and rapidly decay back to "normal levels". On the other hand, the seasonality of temperature creates a seasonally varying average price level. Both spikes and seasonality of power prices are clearly visible in Fig. 1, where we have depicted the spot prices at NordPool in the period April 11997 until July $142000 .{ }^{1}$ The spikes are due to a sudden increase in demand due to a drop in temperature, say, or a fall-out of a major nuclear power plant in Sweden. If a nuclear power plant unexpectedly stops producing, prices will rise to compensate for the drop in supply. This will happen as a rather sharp increase since the supply is inflexible. However, prices will rapidly revert back since more expensive coal and gas fired power plants in Denmark will start operating and demand will decrease.

In the EEX market one has observed negative prices in the spot markets. The reason for this is the priority given to wind generated power in the network. If there is an unexpected increase in wind power generation, other producers may be forced to dump their production. In fact, since it is rather costly to shut down and next ramp up a coal fired power plant, say, it may be better

\footnotetext{
${ }^{1}$ We have selected this rather old period of data for illustration only, since it was a period where prices had a very apparent seasonality and spike pattern.
} 


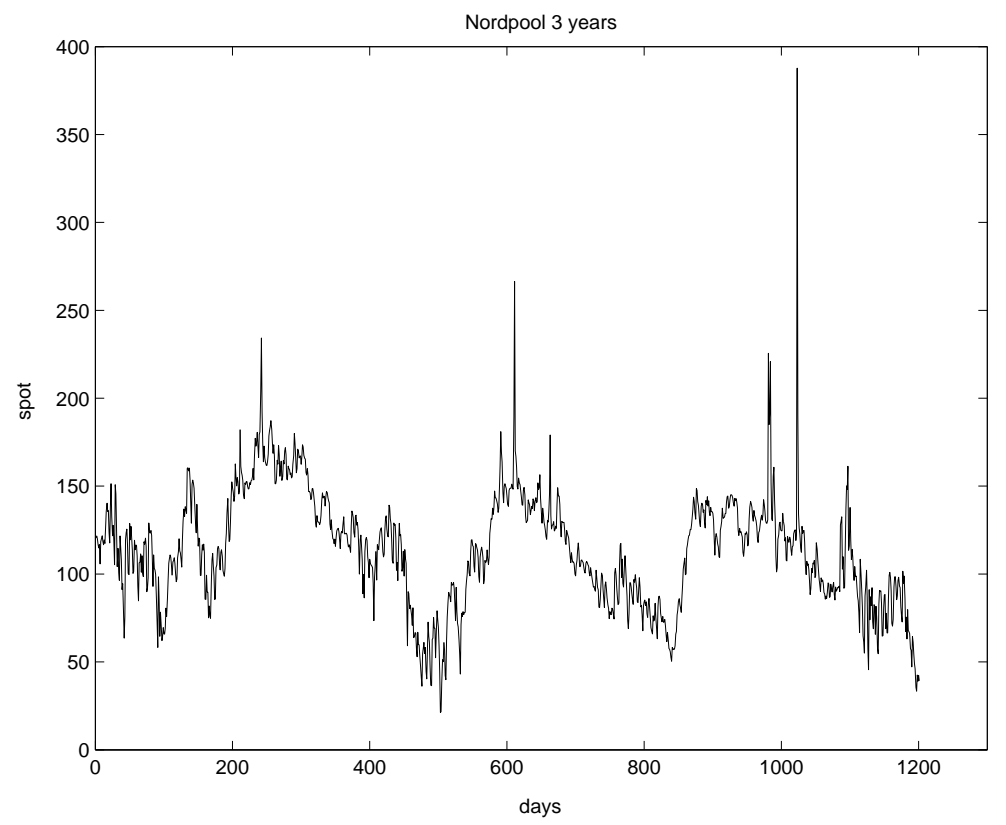

Figure 1. The system price at NordPool from April 11997 untill July 142000. The prices are denominated in NOK per MWh

financially to pay someone to consume the electricity production rather than adjust the power generation.

Coming back to the NordPool market, a decisive factor for the price level is reservoir filling. The amount of rain in the autumn and the snow levels during the winter, determines the production capacity for cheap hydro power, and therefore the prices during winter. A low reservoir filling, for example resulting from a very dry autumn, may lead to excessive prices for power during winter times. This is usually the period when spikes are observed in the NordPool market.

Let us move our attention to the specifics of the electricity market, where we use the NordPool market as the case of discussion. At NordPool, one can trade in spot electricity, forwards and futures contracts as well as plain vanilla call and put options on these. This division into three markets, a spot, forward and option market, is typical for most power markets, as well as other energy markets. The weather market is an obvious exception here, since there is no natural possibility to trade in "spot temperature". The spot market of power is a physical market where one must be either a producer or a consumer/retailer to participate. The two other segments are financially settled, and thus attracts speculators like investment banks, say.

The spot market is auction-based, where producers and consumers are handing in a limited amount of bids the day before. They can bid on buying or selling a certain amount (measured in $\mathrm{MW}=$ mega Watt) of power for a given price transmitted at a given hour the next day. Hence, the bids are for one or more of the 24 hours the next day, giving the volume to buy or sell and the price at which the transaction can take place. The NordPool market is next feeding in all the bids and creating a demand and supply curve for each of the 24 hours the coming day, and by noon the system price is settled for the next day. The system price is the spot price for delivery of $1 \mathrm{MW}$ of electricity in a specific hour. In Fig. 2 we show the hourly system price in Euro per MWh at NordPool on November 23 2011. As is clearly seen, the power is most expensive in the peak hours around 8 in the morning and 6 in the afternoon. The evening and night prices are cheapest. This intra-day price pattern is rather typical. It is noticeable from a modeling point of view that the spot price is a time series, discretized at an hourly level. Furthermore, every day at noon 24 new prices for the next day will be revealed, very much in a similar fashion as a forward market 


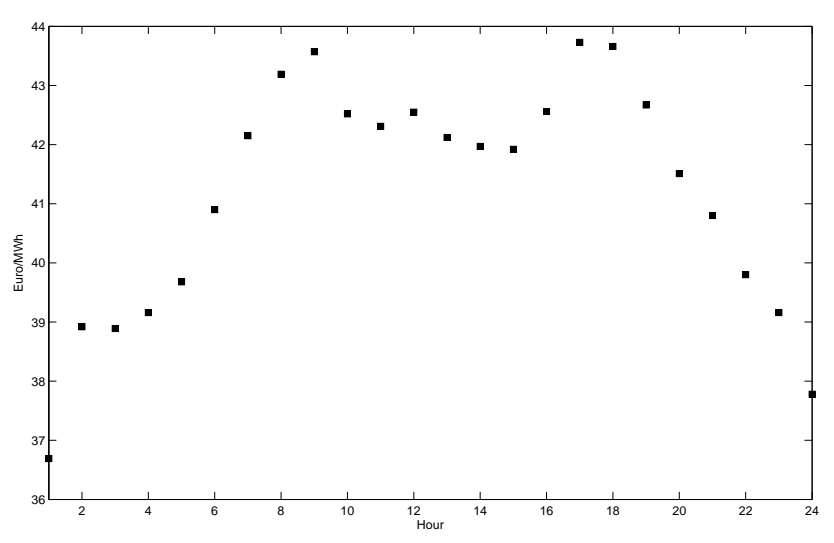

Figure 2. The hourly system price at NordPool on 23 November 2011

is revealing prices for delivery at different times. In some markets, like the UK power market, one trades in half-hourly spot prices.

The hourly system price at NordPool will, however, not be the actual trading price for electricity in the market. Due to congestion, as discussed above, there will be different prices for different areas balancing production, demand and transmission capacity between these areas. These area prices will also be given for each hour, and constitute the actual price for power in that area. In Fig. 3 we have taken a screen shot from the web-page of the NordPool Spot market (see www.nordpoolspot.com), showing the the average system price in the different areas in NordPool on 23 November 2011.

The forward and futures market at NordPool delivers power over a specific period of time. Unlike most other commodity markets that delivers the underlying asset at a specific delivery time, power has to be delivered over a delivery period by its physical nature. Hence, buying a future or forward contract will provide you with power delivered over an agreed period. The NordPool exchange, similar to most other exchanges, is organizing the settlement in financial terms rather than actual physical delivery of power. Thus, buying a forward with delivery over the next month, say, will entitle you to receiving a stream of money equivalent to the spot price at each hour in the next month. If we denote by $S(t)$ the spot price at time $t$, you receive

$$
\sum_{i=1}^{31 \times 24} S\left(t_{i}\right)
$$

where $t_{1}$ is the first hour in the next month, $t_{2}$ the second hour and so on throughout the month (assuming 31 days). The system price (and not the area prices) are used in settling the contract. In return, the owner of the forward will pay a fixed price, called the forward price. It is the market convention to denote this price in terms of Euro per MWh, which is the same denomination as the system price for a given hour. Letting $F\left(t, T_{1}, T_{2}\right)$ be this forward price, with $T_{1}$ and $T_{2}$ being the first and last hour of the coming month, and $t \leq T_{1}$ being the time of entry into the forward contract, we must pay $24 \times 31 \times F\left(t, T_{1}, T_{2}\right)$ in return for receiving (1.1). Hence, we see that a forward contract is essentially a swap of a floating spot price with a fixed price. Often the forwards are called swaps in this market.

Since the forwards and futures contracts are financially settled, one does not need to have any physical capacity for producing or receiving electricity in order to participate in this market segment. Since the NordPool forward and futures are economically equivalent to physically settled contracts, producers and consumers may use them for hedging. But these contracts may also be traded by speculators, for example investment banks and funds, providing more liquidity to the market. This is also what has happened to some extent in the NordPool and EEX markets. In Fig. 4 we have plotted the futures prices for quarterly contracts at the EEX on 23 November 2011. 


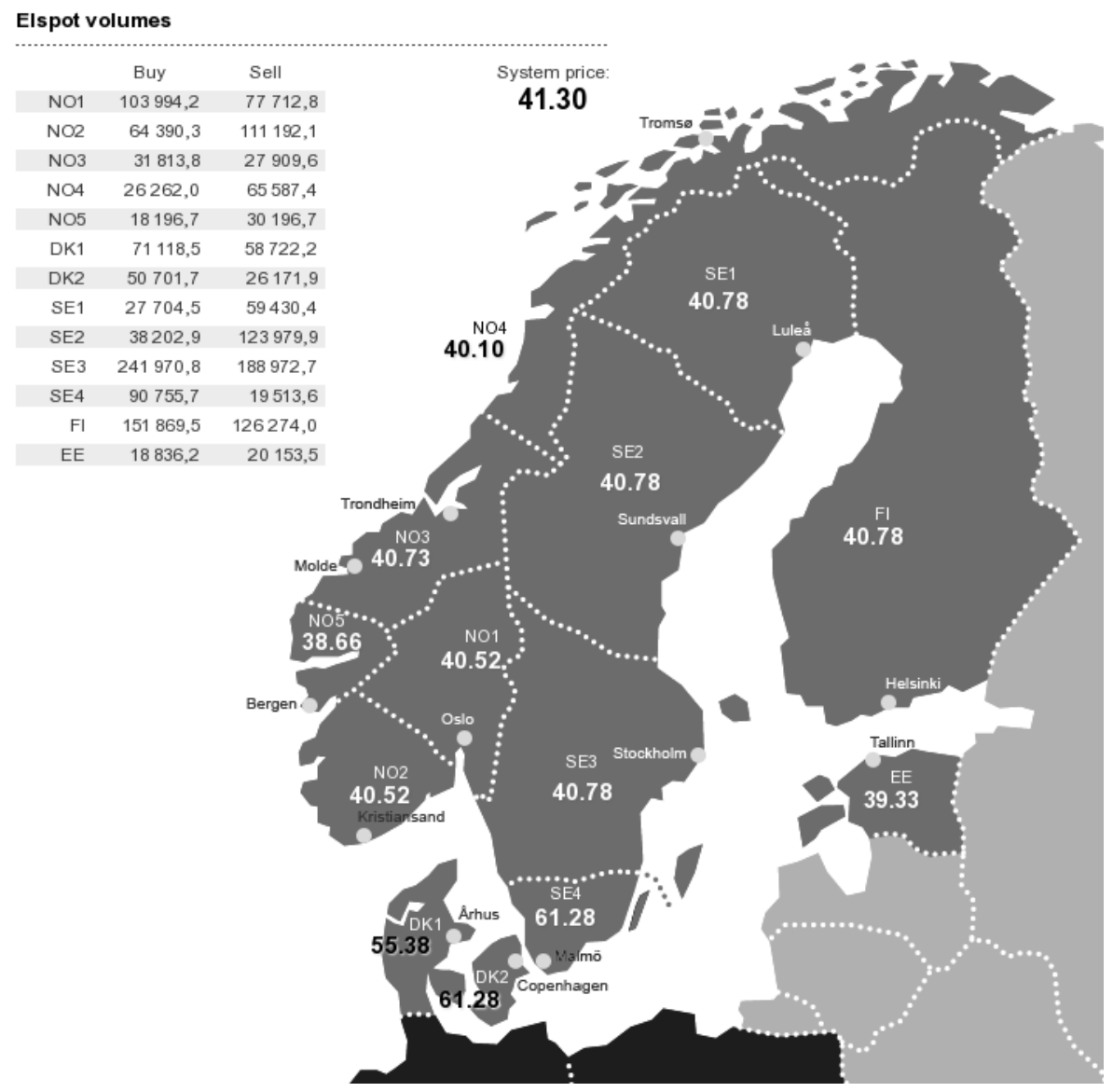

Figure 3. The average area prices at NordPool on 23 November 2011. Prices are denominated in Euros per MWh

As we clearly see, the prices for financial delivery of power in the first and fourth quarters are more expensive than in the second and third. This matches with the winter and summer periods, where one expects cold and warm weather, respectively. The EEX market trades in quarterly futures for two years ahead. The NordPool quarterly forward prices would follow a similar pattern.

NordPool offers both forward and futures contracts. The delivery periods offered in the market ranges from short term daily and weekly delivery, to longer term monthly, quarterly and yearly delivery. Yearly delivery contracts are are offered for up to three years ahead, meaning that you can fix prices for delivery over 2014 today (which is November 2011, at the time of writing), for example. The short term contracts are futures-style, whereas the long-term are forward contracts. The market also distinguishes between peak and base load contracts. Peak load contracts are settled on the system price in peak hours, which are defined from 8 to 20 every working day. These hours are the times when demand is highest. Base load contracts, on the other hand, are settled on all hours in the delivery period. On some of the forward contracts, one can trade in European call and put options. The market for these options have been rather thin.

Although the option trading on the power market is not so active, there exists an abundance of various exotic option contracts traded OTC. So-called swing options, where the holder has the right to buy power at favourable prices, at the same time deciding the amount or volume to be traded, are very popular and appear in many different kinds. For example, the flexible load contracts gives the owner the right to buy electricity at a fixed price in a number of hours over a year. This is an American-style option, where the holder can decide when to exercise within a year, however, having multiple exercise rights. Each time the holder exercises, she also determines 


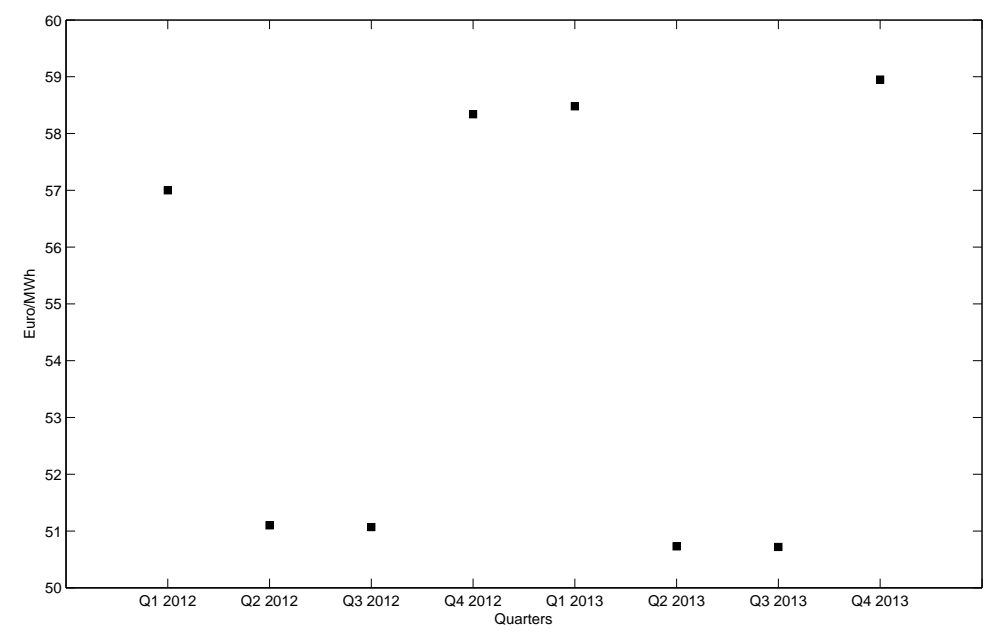

FIgURE 4. Futures prices for contracts delivering over a quarter traded at EEX on 23 November 2011

the volume of power she wants to buy. Hence, in mathematical terms, the owner of the flexible load contract must find an optimal strategy for the exercise times, and an optimal volume control at each time of exercise. Naturally, there are constraints on the volume to take out from the contract at each exercise, as well as a cumulative volume constraint. Such contracts have been analysed in Benth, Lempa and Nilsen [10] using stochastic control theory.

Spread options are very popular tools for managing cross-commodity risk. In the energy markets they appear as spark and dark spread options, say, being options written on the difference between power and gas/coal prices (or, rather the energy equivalent). They may also be combined into swing option like instruments. A typical example is the so-called tolling agreement, which is in effect a virtual power plant. For example, one may get a contract which gives the holder a stream of money every time she decides to produce power, in a virtual power plant using gas as fuel. She decides when to produce, and how much within certain boundaries. When producing, she will receive the spot price of power, in return to paying the spot price of gas. Such options can also be used to value projects of building a gas-fired power plant. Althoug most spread options are traded OTC, there exists a market for spreads between different refined oil products at NYMEX, as well as contracts on the difference between area prices in the NordPool market. The latter is called Contracts-for-Difference (CfD), and are futures written on the price spread between two areas. We refer to Carmona and Durrleman [14] for an extensive discussion and analysis of spread options in energy markets, and Eydeland and Wolyniec [15] for other exotic options.

There exist many other exotic options in the energy markets, like for example various averagetype options and so-called quanto options. The latter are options written on an energy like gas or power, paying out in a call or put fashion. However, the payout is triggered by a weather index, say. For example, one may have a call option on the gas price, which is nulled if a temperature index falls within certain bounds. The latter may provide a control of risk towards demand, while the call structure provides a hedge against high prices of gas. Such products provide then a hedge towards volume risk for the participants in the energy market.

\section{Stochastic Modeling OF ENERGy MARKETS}

We now outline some approaches to stochastic modelling of energy prices, without any intention to be exhaustive in our presentation. The purpose of this subsection is more to create a starting point to the topics presented in these lecture notes, namely cross-commodity and stochastic volatility modelling. 
As the basic model for stock prices is the geometric Brownian motion, the Schwartz dynamics (see Schwartz [26]) is the canonical model in energy (and commodity) markets. Suppose that the spot price follows the stochastic process

$$
S(t)=\Lambda(t) \exp (X(t)),
$$

for $t \geq 0$, where $\Lambda(t)$ is a positive deterministic function modeling the average spot price, also called the seasonality, and $X(t)$ follows the Ornstein-Uhlenbeck (OU) process

$$
d X(t)=-\alpha X(t) d t+\sigma d B(t) .
$$

Here, $\alpha$ and $\sigma$ are positive constants and $B(t)$ is a Brownian motion defined on a complete filtered probability space $\left(\Omega, \mathcal{F},\left\{\mathcal{F}_{t}\right\}_{t \geq 0}, P\right)$. We note that $\ln S(t)$, the logarithmic spot price, will mean revert towards its mean $\ln \Lambda(t)$, at a speed $\alpha$ and volatility given by $\sigma$. A straightforward application of Itô's Formula, assuming $\Lambda(t)$ being differentiable, yields the dynamics

$$
d S(t)=\left(\frac{\Lambda^{\prime}(t)}{\Lambda(t)}+\alpha \ln \Lambda(t)+\frac{1}{2} \sigma^{2}-\alpha \ln S(t)\right) S(t) d t+\sigma S(t) d B(t) .
$$

Thus, the Schwartz dynamics is a "geometric Brownian motion" with a state-dependent drift which is mean-reverting to a seasonal level.

In our survey on energy markets, we saw that the spot prices of electricity were discrete, quoting only prices for hourly delivery. A continuous-time model may seem inappropriate in this context. However, it is rather standard to make such an approximation. One may view the continuous price dynamics as an unobserved price for immediate delivery of electricity, and the actual hourly spot price are simply observations of this. There is a small problem with the filtration using such a view, since the 24 hourly prices are settled the day before, and not according to a stream of information arriving continuously over the day. On the other hand, it is very practical to have a continuous-time model for the spot price dynamics, as one is interested to derive forward prices based on this. The forward prices evolve in a continuous-time market, and not at discrete hourly times.

The model in (2.1)-(2.2) has been suggested for NordPool spot prices by Lucia and Schwartz [22]. In [22], they calibrated this model to spot price data, but also extended it by introducing a second factor driving the spot price. They considered the model

$$
S(t)=\Lambda(t) \exp (X(t)+Y(t))
$$

for a non-stationary term $Y(t)=\mu t+\eta W(t), W(t)$ being a correlated Brownian motion (see Schwartz and Smith [27] for a first application of this model to oil markets). In their paper, Lucia and Schwartz [22] made an extensive study of both spot and forward price modeling in the NordPool market.

The models suggested by Schwartz and co-authors above are driven by Brownian motion, and unlikely to create the large price spikes that one observes in power markets. Also in the gas market one may observe large price increases that may be attributed as spikes, being a result of increase of demand and low storage. A natural way to model spikes is to apply Lévy processes, which may produce a sudden increase of the price from a large upward jump. For example, we may substitute the Brownian motion $B(t)$ in $(2.2)$ by a Lévy process $L(t)$. The speed of mean reversion $\alpha$ will then make sure that a large positive jump in $L(t)$, is followed by an exponentially fast decrease in prices. Letting the speed of mean reversion be sufficiently fast, we can obtain price paths with spikes, a feature which is typically observed in real power price data. Since Lévy processes are rather flexible, one may combine big jumps with many small, and even include a Brownian motion. Alternatively, one may separate the spike behaviour with the "normal variations" of prices, using $Y(t)$ as an OU process driven by Brownian motion rather than a drifted Brownian motion. The choices are many, and the model must be selected by properties of the data which vary considerably between markets. We refer to Benth et al. [4] for a thorough analysis of multi factor models.

There is a debate whether power spot prices are stationary or not. The two-factor model suggested by Lucia and Schwartz [22] above is clearly non-stationary, but letting $Y(t)$ be an OU-process creates a stationary model. For example, in Barndorff-Nielsen et al [1], one finds a very good fit for a stationary one-factor dynamics using the general class of Lévy semistationary 
(LSS) models. LSS models generalize OU processes, and offer a great deal of flexibility to capture the probabilistic properties of spot price data. An unfortunate effect of using stationary spot price models is that the forward prices (theoretically) become constant in the long end of the market. This is not a property one observes for actual forward prices. This could suggest that non-stationary models are more appropriate, or that the connection between spot and forward prices are far more complex in power markets than traditional modelling and pricing suggests (see Benth et al. [5] for a discussion and an equilibrium approach to power forward pricing).

In the EEX market we may have negative prices for the electricity spot. In fact, the NordPool market also allows negative prices. The Schwartz model (2.1) is on an exponential form, yielding positive prices at all times. An alternative specification could be to state the spot prices directly as a one or two factor model, like for example

$$
S(t)=\Lambda(t)+X(t)+Y(t) .
$$

As it turns out, such a model may succesfully calibrate the market data. Benth et al. [8] has estimated such a model to EEX spot prices, using a stable Lévy process driving a CARMA model $X(t)$, and a non-stationary Lévy processes to model $Y(t)$. A CARMA model is a continuous-time autoregressive moving average process, being a specific class of multi-dimensional OU-processes. Apart from allowing for negative prices, such arithmetic models are useful when pricing power forward contracts (which is done in Benth et al. [8]).

In these lecture notes we want to investigate stochastic volatility in a class of spot price models based on the Schwartz dynamics. Leaving aside the issue of jumps, we consider a two-factor model where the stochastic volatility (SV) is driven by a superposition of subordinators, called the Barndorff-Nielsen and Shephard (BNS) SV model. The dynamics generalizes the simpler onefactor stochastic volatility model proposed and analysed by Benth [3], which was fitted gas prices in the UK. In Hikspoors and Jaimungal [19], various stochastic volatility models are analysed in the context of energy.

We will also consider a cross-commodity spot model, where each commodity (or energy) is modelled as a two-factor process. The aim is to price spread options and to analyse some effects of the dependency structure between the two commodities. Although our models are rather simple and specific, we apply them as cases to illustrate some of the main issues and challenges in mathematical finance applied to energy markets.

In power markets, one cannot trade in the underlying spot price of electricity since it is nonstorable, and thus one cannot perform a buy-and-hold strategy to hedge a forward position. The notion of convenience yield does not make sense either (see Geman [17]). Thus, the relationship between the the spot and forwards in power markets is an open question with (yet) no clear answer. What we do know, however, is that the dynamics of the forward price has to be a (local) martingale under some pricing measure $Q \sim P$ in order to ensure an arbitrage-free dynamics of the forward price. Since the underlying spot is not tradeable in the financial sense of the word, such a pricing measure does not have to be a (local) martingale measure for the spot price dynamics. A forward contract with delivery of power at time $T$ and forward price $f(t, T)$ agreed at time $0 \leq t \leq T$, will yield a payoff $f(t, T)-S(T)$ to the seller. From the arbitrage theory for pricing derivatives, we reach the definition of the forward price with respect to the measure $Q$ as

$$
f(t, T)=\mathbb{E}_{Q}\left[S(T) \mid \mathcal{F}_{t}\right]
$$

since the investment costs are zero. A forward contract delivering over $\left[T_{1}, T_{2}\right]$ will have a forward price $F\left(t, T_{1}, T_{2}\right)$ naturally defined as

$$
F\left(t, T_{1}, T_{2}\right)=\mathbb{E}_{Q}\left[\frac{1}{T_{2}-T_{1}} \int_{T_{1}}^{T_{2}} S(T) d T \mid \mathcal{F}_{t}\right]
$$

where we use the approximation that the forward delivers continuously over the delivery period rather than at the discrete hours. Of course, we need to impose certain integrability conditions on the spot price dynamics under $Q$ in order to make these definitions well-posed. 
Interchanging expectation and integration leads to the equation

$$
F\left(t, T_{1}, T_{2}\right)=\frac{1}{T_{2}-T_{1}} \int_{T_{1}}^{T_{2}} f(t, T) d T,
$$

which lends itself to the obvious interpretation of a power forward as a stream of fixed-delivery forwards. In many cases, it is possible to derive the price $f(t, T)$ for a given pricing measure $Q$ rather explicitly, but there is no analytical formula for the integral defining $F\left(t, T_{1}, T_{2}\right)$. This is the case for exponential models of the type in (2.1). Hence, one must perform numerical integration in order to obtain the prices of power forwards. Recalling that the electricity spot prices are settled on an hourly resolution, a natural numerical integration scheme would be a Riemann sum over $f\left(t, T_{i}\right)$, where $T_{i}$ are the different hours of the delivery period. In fact, this would point back to the very definition of a forward contract with delivery period in the electricity market, where in practise the settlement is on the hourly spot prices in the delivery period.

Using arithmetic models of the type (2.5) will in many interesting situations give analytic power forward prices $F\left(t, T_{1}, T_{2}\right)$, at least up to knowledge of the characteristics of the driving noise processes of the factors. This is an attractive aspect of arithmetic models, paving the way for pricing of options on power forwards using Fourier methods which are far more efficient than Monte Carlo simulation, say.

Of course, to pin down the right forward price requires a specification of the "pricing measure $Q$. This is typically done by choosing a parametric class of measures using Girsanov or Esscher transform (see Benth et al. [4]). From this, one may be able to compute theoretical prices, which next can be fitted to observed ones in order to estimate the parameters in the measure transform. This procedure is targeted to explain the risk premium in the market, defined as the difference between forward prices and the predicted spot:

$$
R\left(t, T_{1}, T_{2}\right)=F\left(t, T_{1}, T_{2}\right)-\mathbb{E}\left[\frac{1}{T_{2}-T_{1}} \int_{T_{1}}^{T_{2}} S(T) d T \mid \mathcal{F}_{t}\right] .
$$

Empirical studies of the risk premium in power markets reveal quite a complex behaviour (see Benth et al. [5] for a discussion and references). In commodity markets, one usually expects the risk premium to be negative, a result of hedging pressure from producers accepting to pay a premium to the speculators for locking in prices of their commodities. This is usually referred to as normal backwardation in the market. However, in power markets we may encounter positive risk premia. Such positive premia can be found in the short end of the forward market, that is, for contracts which are close to delivery and with a relatively short delivery period. The reason for this is that the consumers want to lock in prices to hedge the spike risk. The producers are of course not afraid of excessively high prices, but this may be harmful to retailers which may be engaged in fixed-price contracts with their clients. The producers want to hedge using longer term contracts, which shows up as a negative premium in the long end of the forward market. Hence, the picture we see in power markets is a risk premium which changes sign from positive to negative as a function of time to delivery. We refer to Benth et al. [8] for a confirmation of this in the EEX market based on a spot price modeling approach, and to Benth et al. [5] for a simple equilibrium model explaning this along with an empirical case at the EEX. Benth and Sgarra [12] show that the change in sign of the risk premium may possibly be explained by a seasonality in the occurence of spikes.

The Heath-Jarrow-Morton (HJM) approach in interest rate theory suggests to model the forward rates directly rather than via the short rate of interest (see Heath, Jarrow and Morton [18]). This idea was adopted to power markets by Benth and Koekebakker [9], where forward prices are modelled directly rather than via spot prices. The main challenge with the HJM methodology in power markets is the inclusion of a delivery period in the dynamics.

As we have discussed several times already, forward contracts in power markets deliver over various periods. These may in fact be overlapping. In the NordPool market, one can trade in contracts for delivery in the months January, February and March, say, but at the same time one can enter a contract delivering over the first quarter of the year. Of course, the three monthly contracts overlap completely with the quarterly contract. If $T_{i}, i=0,1,2,3$ are the first of each 
of the months January, February, March and April, we must have that the forward prices satisfy the condition

$$
F\left(t, T_{0}, T_{3}\right)=\sum_{i=0}^{2} \frac{T_{i+1}-T_{i}}{T_{3}-T_{0}} F\left(t, T_{i}, T_{i+1}\right),
$$

in order to avoid arbitrage opportunities between the four contracts. If, in an HJM approach, we insist on specifying a model of $F\left(t, T_{s}, T_{e}\right)$ for all possible delivery periods $\left[T_{s}, T_{e}\right]$, with $0 \leq t \leq$ $T_{s}<T_{e}$, we are led to the no-arbitrage condition (see Benth and Koekebakker [9])

$$
F\left(t, T_{s}, T_{e}\right)=\frac{1}{T_{e}-T_{s}} \int_{T_{s}}^{T_{e}} F(t, T, T) d T .
$$

This condition comes in addition to the martingale restriction on the dynamics of $F\left(t, T_{s}, T_{e}\right)$ under the pricing measure $Q$. Since $F(t, T, T)$ is the forward price of a contract delivering at time $T$, we are in a situation where any model of $F\left(t, T_{s}, T_{e}\right)$ is brought back to a model for $f(t, T)$. Hence, it is natural to model fixed-delivery power forward prices $f(t, T)$, although these do not exist in the market (see Benth et al. [4] for a discussion on this, both analytically and empirically).

Alternatively, one may take a LIBOR modeling point of view (see for example Brigo and Mercurio [13]), and simply focus on the traded delivery periods, that is, to model only the forwards which are traded in the market. We first single out the smallest delivery periods, and model these exclusively. For example, going back to the case above, we model only the forward price dynamics of the three monthly contracts, and let the quarterly contract has a forward price given by (2.10). This program was proposed and studied in the context of the NordPool market in Benth and Koekebakker [9], and further extended in Benth et al. [4].

\section{A multi-Factor Stochastic VOlatility MODEL FOR ENERGy PRICES}

Let us consider the question of modelling the spot price dynamics of an energy commodity. We denote the price at time $t \geq 0$ by $S(t)$, and choose to model the dynamics in continuous time although it may be discrete in some markets like for example electricity as discussed above. We focus our attention on a single market, and propose a multi-factor model which accounts for many of the stylized facts observed in power and energy markets. Inspired by Hikspoors and Jaimungal [19], we analyse a spot price model of exponential type which incorporates stochastic volatility, and where prices are mean-reverting towards a stochastic level.

To be slightly more specific, we suppose that the spot price on logarithmic scale is given as an Ornstein-Uhlenbeck process reverting towards a stochastic level, and driven by a Brownian motion scaled by a stochastic volatility. The stochastic volatility follows the so-called Barndorff-Nielsen and Shephard (BNS) model, see [2]. The level towards which the log-spot prices are reverting will be assumed to be again an Ornstein-Uhlenbeck dynamics. Hikspoors and Jaimungal [19] assume a Brownian-based dynamics of the stochastic volatility, including for example the Heston model, and focus on an asymptotic analysis of derivatives. We now introduce our model rigourously, starting out by defining the stochastic volatility part.

We denote by $L_{j}, j=1, \ldots, n, n$ independent subordinator processes, that is, increasing Lévy processes. We choose to work with the RCLL version of the $L_{j}$ 's. Define for $j=1, \ldots, n$ the Ornstein-Uhlenbeck process

$$
d Y_{j}(t)=-\lambda_{j} Y_{j}(t) d t+d L_{j}(t),
$$

where $\lambda_{j}>0$ is constant. The Lévy measure of $L_{j}$ is denoted $\ell_{j}$. Let $w_{j}>0$ and $w_{1}+\ldots+w_{n}=1$, and define a volatility process $\sigma(t)$ by

$$
\sigma^{2}(t)=\sum_{j=1}^{n} w_{j} Y_{j}(t)
$$

Note that since the $L_{j}$ 's are subordinators, it follows that $Y_{j}(t)$ are non-negative for all $j=1, \ldots, n$, and thus $\sigma^{2}(t)$ is non-negative as well. Therefore, $\sigma(t)$, the square-root of $\sigma^{2}(t)$, is well defined. 
We shall assume that the subordinators are driftless, that is, that $L_{j}(1)$ have cumulant functions given by

$$
\psi_{j}(\theta):=\ln \mathbb{E}\left[\exp \left(\mathrm{i} \theta L_{j}(1)\right)\right]=\int_{0}^{\infty}\left\{\mathrm{e}^{\mathrm{i} \theta z}-1\right\} \ell_{j}(d z)
$$

for $j=1, \ldots, n$.

Note that we have a constant volatility process $\sigma(t)$ whenever $\lambda_{j}=0$ and $L_{j}=0$ for all $j$. If only the latter holds, the volatility becomes deterministic, converging to zero with time. To account for possible seasonal effects one may allow for time-dependent coefficients $w_{j}$ and $\lambda_{j}$. However, we shall not consider this case, but restrict our attention to constant coefficients.

In our spot price model we suppose that the seasonal level is modelled by a bounded and measurable function $\Lambda:[0, \infty) \mapsto \mathbb{R}_{+}$. In case there is no seasonality, $\Lambda(t)$ is simply a constant, usually put equal to 1 .

Define the spot price as

$$
S(t)=\Lambda(t) \exp (X(t))
$$

with

$$
\begin{aligned}
d X(t) & =(Z(t)-\alpha X(t)) d t+\sigma(t) d B_{1}(t) \\
d Z(t) & =(\mu-\beta Z(t)) d t+\eta d B_{2}(t) .
\end{aligned}
$$

The Brownian motions $B_{1}$ and $B_{2}$ are correlated by a factor $\rho$, and independent of the subordinators $L_{j}, j=1, \ldots, n$. This means that the volatility process $\sigma(t)$ is independent of the stochastic drivers of the mean level and the log-spot price. The deseasonalized log-spot prices are meanreverting like in the one-factor model, however, now towards a stochastic mean, which again is a mean reverting process. From an applied point of view, it is natural to imagine that the stochastic mean is slowly mean reverting, while the prices themselves mean revert at a higher speed. Geman [17] argues for such a dynamics for oil prices using a Cox-Ingersoll-Ross model for $\sigma^{2}(t)$.

In the literature on energy spot price models (see Ch. 3 in Benth et al. [4] and the references therein) factor models are usually stated directly as a sum of stochastic processes. A simple two-factor model for the spot price on exponential form is given as

$$
S(t)=\Lambda(t) \exp \left(X_{1}(t)+X_{2}(t)\right)
$$

with

$$
d X_{i}(t)=-\alpha_{i} X_{i}(t) d t+\sigma_{i} d B_{i}(t)
$$

for $i=1,2$. Letting $X(t):=X_{1}(t)+X_{2}(t)$, we find the dynamics of $X(t)$ to be

$$
d X(t)=\left(\left(\alpha_{1}-\alpha_{2}\right) X_{2}(t)-\alpha_{1} X(t)\right) d t+\sigma_{1} d B_{1}(t)+\left(\alpha_{1}-\alpha_{2}\right) \sigma_{2} d B_{2}(t) .
$$

Hence, by identifying $Z(t)=\left(\alpha_{1}-\alpha_{2}\right) X_{2}(t)$ we see that we recover our mean-reversion model with a stochastic level in (3.4). We remark in passing that the two-factor model of Lucia and Schwartz [22] assumes the factor $X_{2}(t)$ to be non-stationary. We may incorporate that case by supposing $X_{2}(t)$ having zero mean-reversion, $\alpha_{2}=0$. This would correspond to a stochastic meanlevel being non-stationary, that is, equivalent to letting $\beta=0$ in the process $Z(t)$ in the model (3.4). The Lucia and Schwartz model was first proposed by Schwartz and Smith [27] for spot oil prices.

In the analysis of our spot price model, we want to derive probabilistic properties like the induced covariance structure, as well as the implied forward prices for contracts written on the spot. In the sequel, we suppose that $\alpha \neq \beta$ to avoid a singular case. Our first result derives the explicit dynamics of $X(t), Z(t)$ and $Y_{j}(t), j=1, \ldots, n$ :

Lemma 3.1. Suppose that $u \mapsto \sigma(u) \exp (-\alpha(u-t))$ is Itô integrable on $u \in[s, t]$ for $s \geq t$. The explicit dynamics of $X(s), Z(s)$ and $Y_{j}(s)$ given $X(t), Z(t)$ and $Y_{j}(t)$ for $s \geq t$ are

$$
\begin{gathered}
X(s)=X(t) \mathrm{e}^{-\alpha(s-t)}+\int_{t}^{s} Z(u) \mathrm{e}^{-\alpha(s-u)} d u+\int_{t}^{s} \sigma(s) \mathrm{e}^{-\alpha(s-u)} d B_{1}(u), \\
Z(s)=Z(t) \mathrm{e}^{-\beta(s-t)}+\frac{\mu}{\beta}\left(1-\mathrm{e}^{-\beta(s-t)}\right)+\int_{t}^{s} \eta \mathrm{e}^{-\beta(s-u)} d B_{2}(u) .
\end{gathered}
$$


and

$$
Y_{j}(s)=Y_{j}(t) \mathrm{e}^{-\lambda_{j}(s-t)}+\int_{t}^{s} \mathrm{e}^{-\lambda_{j}(s-u)} d L_{j}(u) .
$$

Proof. We apply Itô's Formula on the process $\exp (\alpha u) X(u)$ to find

$$
\begin{aligned}
d\left(\mathrm{e}^{\alpha u} X(u)\right) & =\alpha \mathrm{e}^{\alpha u} X(u) d t+\mathrm{e}^{\alpha u} d X(u) \\
& =\mathrm{e}^{\alpha u} Z(u) d u+\sigma(u) \mathrm{e}^{\alpha u} d B_{1}(u) .
\end{aligned}
$$

Integrating from $t$ to $s$ yields the result for $X(s)$. A similar computation shows the result for $Z(s)$. Finally, using the Itô Formula for jump processes (see Ikeda and Watanabe [20]) yields the result for $Y_{j}(s)$.

To this end, define the function

$$
\gamma(u ; \alpha, \beta)=\frac{1}{\alpha-\beta}\left(\mathrm{e}^{-\beta u}-\mathrm{e}^{-\alpha u}\right) .
$$

This function will appear in several places in connection with the analysis of our spot price model. It is simple to observe that $\gamma(u ; \alpha, \beta)$ is a non-negative continuous function, with $\gamma(0 ; \alpha, \beta)=0$ and $\lim _{u \rightarrow \gamma} \gamma(u ; \alpha, \beta)=0$. Furthermore, by a straightforward differentiation, it attains its maximum value at

$$
u^{*}=\frac{\ln \alpha-\ln \beta}{\alpha-\beta} .
$$

The maximal value can be computed to be

$$
\gamma^{*}(\alpha, \beta):=\max _{u \geq 0} \gamma(u ; \alpha, \beta)=\gamma\left(u^{*} ; \alpha, \beta\right)=\frac{\beta^{\beta / \alpha-\beta}}{\alpha^{\alpha / \alpha-\beta}} .
$$

We shall make use of the properties of the function $\gamma$ throughout this Section.

By applying the explicit form of $Z(s)$ in Lemma 3.1, we can derive the following explicit dynamics of $X(s)$ :

Lemma 3.2. The explicit dynamics of $X(s)$ given $X(t)$ for $s \geq t$ can be represented as

$$
\begin{array}{r}
X(s)=X(t) \mathrm{e}^{-\alpha(s-t)}+\left(Z(t)-\frac{\mu}{\beta}\right) \gamma(s-t ; \alpha, \beta)+\frac{\mu}{\beta} \gamma(s-t ; \alpha, 0) \\
+\int_{t}^{s} \sigma(u) \mathrm{e}^{-\alpha(s-u)} d B_{1}(u)+\int_{t}^{s} \eta \gamma(s-u ; \alpha, \beta) d B_{2}(u) .
\end{array}
$$

Proof. From the explicit dynamics of $Z(u)$ given $Z(t), u \geq t$ in Lemma 3.1, we get

$$
\begin{gathered}
\int_{t}^{s} Z(u) \mathrm{e}^{\alpha u} d u=Z(t) \int_{t}^{s} \mathrm{e}^{\alpha u-\beta(u-t)} d u+\int_{t}^{s} \mathrm{e}^{\alpha u} \frac{\mu}{\beta}\left(1-\mathrm{e}^{-\beta(u-t)}\right) d u \\
+\eta \int_{t}^{s} \mathrm{e}^{\alpha u} \int_{t}^{u} \mathrm{e}^{-\beta(u-v)} d B_{2}(v) d u .
\end{gathered}
$$

Applying the stochastic Fubini theorem and the definition of the function $\gamma(u ; \alpha, \beta)$ yield the result.

We continue with analysing the covariance structure of the spot price dynamics. For this study, we must suppose that the log-spot prices have finite variance. By inspection of $X(s)$ in the Lemma above, the log-spot price has finite variance as long as $X(t)$ is of finite variance. But $X(t)$ has finite variance if and only if the stochastic integral with respect to $B_{1}$ has finite variance, since the other stochastic integral is a simple Wiener integral of a deterministic function. By the Itô isometry, we find

$$
\begin{aligned}
\mathbb{E}\left[\left(\int_{0}^{t} \sigma(u) \mathrm{e}^{-\alpha(t-u)} d B_{1}(u)\right)^{2}\right] & =\mathbb{E}\left[\int_{0}^{t} \sigma^{2}(u) \mathrm{e}^{-2 \alpha(t-u)} d u\right] \\
& =\int_{0}^{t} \mathbb{E}\left[\sigma^{2}(u)\right] \mathrm{e}^{-2 \alpha(t-u)} d u
\end{aligned}
$$


But, from the definition of $\sigma^{2}(u)$, we have

$$
\begin{aligned}
\mathbb{E}\left[\sigma^{2}(u)\right] & =\sum_{j=1}^{n} w_{j} \mathbb{E}\left[Y_{j}(u)\right] \\
& =\sum_{j=1}^{n} w_{j} Y_{j}(0) \mathrm{e}^{-\lambda_{j} t}+\sum_{j=1}^{n} w_{j} \mathbb{E}\left[\int_{0}^{t} \mathrm{e}^{-\lambda_{j}(t-u)} d L_{j}(u)\right] .
\end{aligned}
$$

We know from Benth et al. [4] that

$$
\mathbb{E}\left[\int_{0}^{t} \mathrm{e}^{-\lambda_{j}(t-u)} d L_{j}(u)\right]=\mathbb{E}\left[L_{j}(1)\right] \int_{0}^{t} \mathrm{e}^{-\lambda_{j}(t-u)} d u .
$$

Hence, if $L_{j}(1)$ has finite expectation for all $j=1, \ldots, n$, then $X(t)$ has finite variance. From the definition of the cumulant functions of $L_{j}(1)$ in $(3.3), L_{j}(1), j=1, \ldots, n$ have finite expectation if and only if

$$
\int_{0}^{\infty} z \ell_{j}(d z)<\infty, j=1, \ldots, n .
$$

From now on, we suppose that (3.9) holds. Under this condition we have the required Itô integrability in Lemma 3.1 .

In the next proposition we find the covariance between deseasonalized log-spot prices at different time instances.

Proposition 3.3. For $t, \tau>0$, it holds that

$$
\operatorname{Cov}(X(t+\tau), X(t))=a(t) \mathrm{e}^{-\alpha \tau}+b(t) \mathrm{e}^{-\beta \tau},
$$

where

$$
a(t)=\operatorname{Var}(X(t))-b(t), \quad b(t)=\frac{\operatorname{Cov}(Z(t), X(t))}{\alpha-\beta} .
$$

Proof. Since, by Lemma 3.1

$$
X(t+\tau)=X(t) \mathrm{e}^{-\alpha \tau}+\int_{t}^{\tau} Z(s) \mathrm{e}^{-\alpha(t+\tau-s)} d s+\int_{t}^{t+\tau} \sigma(s) \mathrm{e}^{-\alpha(t+\tau-s)} d B_{1}(s)
$$

we find

$$
\operatorname{Cov}(X(t+\tau), X(t))=\mathrm{e}^{-\alpha \tau}\left(\operatorname{Var}(X(t))+\int_{t}^{t+\tau} \mathrm{e}^{-\alpha(t-s)} \operatorname{Cov}(Z(s), X(t)) d s\right)
$$

since $X(t)$ and $\int_{t}^{t+\tau} \sigma(s) \exp (-\alpha(t+\tau-s)) d B_{1}(s)$ are zero correlated due to the independent increment property of Brownian motion. Recalling $Z(s)$ given $Z(t)$ for $s \geq t$ in Lemma 3.1, we have

$$
\operatorname{Cov}(Z(s), X(t))=\mathrm{e}^{-\beta(s-t)} \operatorname{Cov}(Z(t), X(t)) .
$$

since $X(t)$ and $\int_{t}^{s} \eta \exp (-\beta(t-u)) d B_{2}$ are independent. The proposition follows after a straightforward integration.

In the next lemmas we calculate the variance of $X(t)$ and the covariance of $Z(t)$ and $X(t)$, and investigate their asymptotics when time goes to infinity.

Lemma 3.4. It holds that

$$
\begin{gathered}
\operatorname{Var}(X(t))=\int_{0}^{t} \mathbb{E}\left[\sigma^{2}(s)\right] \mathrm{e}^{-2 \alpha(t-s)} d s+2 \rho \frac{\eta}{\alpha-\beta} \int_{0}^{t} \mathbb{E}[\sigma(s)]\left(\mathrm{e}^{-(\alpha+\beta)(t-s)}-\mathrm{e}^{-2 \alpha(t-s)}\right) d s \\
+\frac{\eta^{2}}{(\alpha-\beta)^{2}}\left\{\frac{1}{2 \beta}\left(1-\mathrm{e}^{-2 \beta t}\right)-\frac{2}{\alpha+\beta}\left(1-\mathrm{e}^{-(\alpha+\beta) t}\right)+\frac{1}{2 \alpha}\left(1-\mathrm{e}^{-2 \alpha t}\right)\right\} .
\end{gathered}
$$


Proof. From Lemma 3.1 we compute using the Itô isometry,

$$
\begin{aligned}
\operatorname{Var}(X(t))= & \mathrm{e}^{-2 \alpha t} \operatorname{Var}\left(\int_{0}^{t} Z(s) \mathrm{e}^{\alpha s} d s\right)+2 \mathrm{e}^{-2 \alpha t} \operatorname{Cov}\left(\int_{0}^{t} \sigma(s) \mathrm{e}^{\alpha s} d B_{1}(s), \int_{0}^{t} Z(s) \mathrm{e}^{\alpha s} d s\right) \\
& +\int_{0}^{t} \mathbb{E}\left[\sigma^{2}(s)\right] \mathrm{e}^{-2 \alpha(t-s)} d s .
\end{aligned}
$$

We consider the first two terms. Applying the stochastic Fubini Theorem, it holds that

$$
\begin{gathered}
\int_{0}^{t} Z(s) \mathrm{e}^{\alpha s} d s=\frac{Z(0)}{\alpha-\beta}\left(\mathrm{e}^{(\alpha-\beta) t}-1\right)+\frac{\mu}{\beta}\left\{\frac{1}{\alpha}\left(\mathrm{e}^{\alpha t}-1\right)-\frac{1}{\alpha-\beta}\left(\mathrm{e}^{(\alpha-\beta) t}-1\right)\right\} \\
+\frac{\eta}{\alpha-\beta} \int_{0}^{t} \mathrm{e}^{\alpha u}\left(\mathrm{e}^{(\alpha-\beta)(t-u)}-1\right) d B_{2}(u) .
\end{gathered}
$$

Thus,

$$
\operatorname{Var}\left(\int_{0}^{t} Z(s) \mathrm{e}^{\alpha s} d s\right)=\frac{\eta^{2}}{(\alpha-\beta)^{2}} \int_{0}^{t} \mathrm{e}^{2 \alpha u}\left(\mathrm{e}^{(\alpha-\beta)(t-u)}-1\right)^{2} d u,
$$

which gives us the last term involving $\eta^{2} /(\alpha-\beta)^{2}$ in the expression of $\operatorname{Var}(X(t))$. Finally, by Itô's isometry and the correlation between $B_{1}$ and $B_{2}$, we find that

$$
\operatorname{Cov}\left(\int_{0}^{t} \sigma(s) \mathrm{e}^{\alpha s} d B_{1}(s), \int_{0}^{t} Z(s) \mathrm{e}^{\alpha s} d s\right)=\rho \frac{\eta}{\alpha-\beta} \int_{0}^{t} \mathbb{E}[\sigma(s)] \mathrm{e}^{2 \alpha s}\left(\mathrm{e}^{(\alpha-\beta)(t-s)}-1\right) d s .
$$

Hence, the lemma follows.

The covariance between $Z(t)$ and $X(t)$ is derived next:

Lemma 3.5. It holds that

$\operatorname{Cov}(Z(t), X(t))=\rho \eta \int_{0}^{t} \mathbb{E}[\sigma(s)] \mathrm{e}^{-(\alpha+\beta)(t-s)} d s+\frac{\eta^{2}}{\alpha-\beta}\left\{\frac{1}{2 \beta}\left(1-\mathrm{e}^{-2 \beta t}\right)-\frac{1}{\alpha+\beta}\left(1-\mathrm{e}^{-(\alpha+\beta) t}\right)\right\}$.

Proof. Using the expression for

$$
\int_{0}^{t} Z(s) \mathrm{e}^{\alpha s} d s
$$

calculated in the proof of Lemma 3.4, it follows that

$$
\begin{aligned}
\operatorname{Cov}(Z(t), X(t))= & \frac{\eta^{2}}{\alpha-\beta} \mathrm{e}^{-(\alpha+\beta) t} \operatorname{Cov}\left(\int_{0}^{t} \mathrm{e}^{\beta s} d B_{2}(s), \int_{0}^{t} \mathrm{e}^{\alpha s}\left(\mathrm{e}^{(\alpha-\beta)(t-s)}-1\right) d B_{2}(s)\right) \\
& +\eta \mathrm{e}^{-(\alpha+\beta) t} \operatorname{Cov}\left(\int_{0}^{t} \mathrm{e}^{\beta s} d B_{2}(s), \int_{0}^{t} \sigma(s) \mathrm{e}^{\alpha s} d B_{1}(s)\right) \\
= & \frac{\eta^{2}}{\alpha-\beta} \mathrm{e}^{-(\alpha+\beta) t} \int_{0}^{t} \mathrm{e}^{(\alpha+\beta) s}\left(\mathrm{e}^{(\alpha-\beta)(t-s)}-1\right) d s \\
& +\rho \eta \mathrm{e}^{-(\alpha+\beta) t} \int_{0}^{t} \mathbb{E}[\sigma(s)] \mathrm{e}^{(\alpha+\beta) s} d s,
\end{aligned}
$$

where we have used the Itô isometry in the last equality. The lemma follows after a straightforward integration.

Notice that when $\rho=0$, that is, when the noises of $X$ and $Z$ are independent, the term involving $\mathbb{E}[\sigma(s)]$ in $\operatorname{Var}(X(t))$ and $\operatorname{Cov}(Z(t), X(t))$ disappears.

We want to show that $X(t)$ has a "stationary" autocorrelation function: observe from Sato [25], Thm. 17.5, that $\sigma^{2}(t)$ has a stationary distribution function with cumulant

$$
\psi_{\infty}(\theta)=\sum_{j=1}^{n} \int_{0}^{\infty} \psi_{j}\left(\theta \mathrm{e}^{-\lambda_{j} u}\right) d u
$$

as long as the condition

$$
\int_{2}^{\infty} \ln z \ell_{j}(d z)<\infty
$$


holds for each subordinator $L_{j}, j=1, \ldots, n$. But for $z>2, \ln z \leq z$, and by our standing assumption of finite expecation of the $L_{j}(1)$, this condition holds true. Hence, the stochastic volatility process $\sigma^{2}(t)$ has a stationary distribution when $t$ tends to infinity, which will be supported on the positive real line. Hence, both $\mathbb{E}\left[\sigma^{2}(t)\right]$ and $\mathbb{E}[\sigma(t)]=\mathbb{E}\left[\sqrt{\sigma^{2}(t)}\right]$ will have limits being strictly positive when $t$ tends to infinity. From the explicit expressions of $Y_{j}$ in Lemma 3.1, it holds in particular that

$$
\lim _{t \rightarrow \infty} \mathbb{E}\left[\sigma^{2}(t)\right]=\sum_{j=1}^{n} \frac{1}{\lambda_{j}} \int_{0}^{\infty} z \ell_{j}(d z) .
$$

If $\xi>0$, we therefore find by L'Hopitals rule that

$$
\lim _{t \rightarrow \infty} \mathrm{e}^{-\xi t} \int_{0}^{t} \mathbb{E}\left[\sigma^{2}(s)\right] \mathrm{e}^{\xi s} d s=\lim _{t \rightarrow \infty} \frac{\mathbb{E}\left[\sigma^{2}(t)\right] \mathrm{e}^{\xi t}}{\xi \mathrm{e}^{\xi t}}=\sum_{j=1}^{n} \frac{1}{\xi \lambda_{j}} \int_{0}^{\infty} z \ell_{j}(d z) .
$$

Similarly,

$$
\lim _{t \rightarrow \infty} \mathrm{e}^{-\xi t} \int_{0}^{t} \mathbb{E}[\sigma(s)] \mathrm{e}^{\xi s} d s=c / \xi
$$

where $c$ is the limit of $\mathbb{E}[\sigma(t)]$. In conclusion, both $\operatorname{Var}(X(t))$ and $\operatorname{Cov}(Z(t), X(t))$ have limits when $t$ goes to infinity. Therefore, we see from Prop. 3.3 that

$$
\lim _{t \rightarrow \infty} \operatorname{Corr}(X(t+\tau), X(t))=c_{1} \mathrm{e}^{-\alpha \tau}+c_{2} \mathrm{e}^{-\beta \tau},
$$

for two positive constants $c_{1}, c_{2}$ such that $c_{1}+c_{2}=1$. The autocorrelation function of the deseasonalized log-spot prices is thus given as a sum of two exponentially decaying functions. This can be utilized in calibration of the spot model, since we can find the speeds of mean-reversion $\alpha$ and $\beta$ by minimizing the distance between the theoretical and empirical autocorrelation functions. The characteristics of the stochastic volatility $\sigma(t)$ and its square enter in $c_{1}$ and $c_{2}$, that is, in the weighting of the two exponential functions.

We observe by Lemma 3.1 that for a small $\Delta>0$, it approximately holds

$$
\mathrm{e}^{\alpha \Delta} X(t+\Delta)-X(t) \approx Z(t) \Delta+\sigma(t) \Delta B_{1}(t),
$$

with $\Delta B_{1}(t)=B_{1}(t+\Delta)-B_{1}(t)$. If we suppose that we can observe the mean-level process $Z(t)$ (using for example filtering), we can find observations of the residual process $\sigma(t) \Delta B_{1}(t)$ by the relation

$$
\sigma(t) \Delta B_{1}(t) \approx \mathrm{e}^{\alpha \Delta} X(t+\Delta)-X(t)-Z(t) \Delta .
$$

Note that $X(t)=\ln S(t)-\ln \Lambda(t)$, and therefore $X(t)$ is directly observable from the spot prices given that we know the seasonality function $\Lambda(t)$. We have, by independence between $\sigma(t)$ and $B_{1}(t)$, that $\sigma(t) \Delta B_{1}(t)$ is a variance-mixture model, where $\sigma(t) \Delta B_{1}(t)$ conditioned on $\sigma^{2}(t)$ will be normally distributed with zero mean and variance $\sigma^{2}(t) \Delta$. Taking $\sigma^{2}(t)$ stationary, we can obtain a rich class of heavy-tailed distributions for these residuals, including for example the normal inverse Gaussian distribution. We refer to Barndorff-Nielsen and Shephard [2] for an in-depth analysis of this, where methods for estimating the factors $Y_{j}$ of the stochastic volatility model are presented and discussed.

3.1. Forward prices. Recall from Section 2 that $f(t, T)$ denotes the forward price at time $t \geq 0$ of a contract delivering the energy spot at time $T$, where $0 \leq t \leq T$. As argued in Section 2 , the arbitrage theory of mathematical finance tells us that the process $t \mapsto f(t, T)$ must be a martingale with respect to some equivalent probability $Q$. This led to the defintion

$$
f(t, T)=\mathbb{E}_{Q}\left[S(T) \mid \mathcal{F}_{t}\right] .
$$

In the standard pricing theory, the underlying asset of the forward is also a tradeable asset, and $Q$ is therefore a martingale measure for $S$. However, in energy markets, trading constraints like storage and transportation of the energy (in case of gas and oil), or no-storage posssibilites at all (in the electricity case) create an incomplete market where the buy-and-hold hedging strategy in the spot cannot be applied. Hence, the measure $Q$ does not need to be a martingale measure for 
$S$. One typically lets $Q$ be part of the modelling, and chooses it in a parametric class of equivalent probability measures, using (3.11) as the definition of the forward price dynamics.

Before deriving expressions for the forward price based on our spot price model, we look at a parametric class of equivalent probability measures $Q$ which changes the Brownian motions in the spot model by a Girsanov transform. To simplify our considerations, we have chosen not to consider any measure change of the subordinators driving the stochastic volatility process $\sigma(t)$.

Represent the Brownian motion $B_{2}$ as

$$
B_{2}(t)=\rho B_{1}(t)+\sqrt{1-\rho^{2}} U(t),
$$

with $U$ being a Brownian motion independent of $B_{1}$. Next, let $\theta_{1}, \theta_{2}$ be two constants which we will call the market prices of risk, and define the adapted stochastic processes

$$
\begin{aligned}
& \widetilde{\theta}_{1}(t) \triangleq \frac{\theta_{1}}{\sigma(t)} \\
& \widetilde{\theta}_{2}(t) \triangleq \frac{\theta_{2}-\rho \eta \widetilde{\theta}_{1}(t)}{\eta \sqrt{1-\rho^{2}}} .
\end{aligned}
$$

Then, by the Girsanov Theorem, it is easy to see that there exists a probability $Q$ such that $W_{1}$ and $V$ defined as

$$
\begin{aligned}
d B_{1}(t) & =\widetilde{\theta}_{1}(t) d t+d W_{1}(t) \\
d U(t) & =\widetilde{\theta}_{2}(t) d t+d V(t),
\end{aligned}
$$

are two independent Brownian motions on a finite time interval $\left[0, T_{\max }\right]$ for any $T_{\max }<\infty$. Indeed, the Novikov condition for Girsanov's Theorem is satisfied since $\sigma(t)$ is bounded from below by a weighted sum of exponential functions: by Lemma 3.1 we have $Y_{j}(t) \geq Y_{j}(0) \exp \left(-\lambda_{j} t\right)$ for $j=1, \ldots, n$. Hence,

$$
\sigma^{2}(t) \geq \sum_{j=1}^{n} w_{j} Y_{j}(0) \mathrm{e}^{-\lambda_{j} t}
$$

Note that the characteristics of $Y_{j}$ remain unaltered under the probability $Q$. This means that we suppose the market price of volatility risk be zero, although there are empirical studies showing that such a risk is present in energy markets (see Trolle and Schwartz [28]). There exist many other risk neutral probabilities that can be used, which can account for such risk premia as well. We refer to the class of Esscher transformed measures that will be introduced later in Section 4 as a possible choice.

here we focus on the standard class of measure change frequently used in commodity analysis.

We find the $Q$-dynamics of $X$ and $Z$ to be,

$$
\begin{aligned}
d X(t) & =\left(\theta_{1}+Z(t)-\alpha X(t)\right) d t+\sigma(t) d W_{1}(t) \\
d Z(t) & =\left(\mu+\theta_{2}-\beta Z(t)\right) d t+\eta d W_{2}(t),
\end{aligned}
$$

where $W_{2} \triangleq \rho W_{1}+\sqrt{1-\rho^{2}} V$ is a $Q$-Brownian motion correlated with $W_{1}$ by the factor $\rho$. Computing as in Lemma 3.2, we find for $t \leq s \leq T_{\max }$

$$
\begin{gathered}
X(s)=X(t) \mathrm{e}^{-\alpha(s-t)}+\left(Z(t)-\frac{\mu+\theta_{2}}{\beta}\right) \gamma(s-t ; \alpha, \beta)+\left(\theta_{1}+\frac{\mu+\theta_{2}}{\beta}\right) \gamma(s-t ; \alpha, 0) \\
+\int_{t}^{s} \sigma(u) \mathrm{e}^{-\alpha(s-u)} d W_{1}(u)+\eta \int_{t}^{s} \gamma(s-u ; \alpha, \beta) d W_{2}(u),
\end{gathered}
$$

Before moving on to compute the forward price, we must ensure that $S(T)$ is integrable with respect to the probability $Q$. The integrability of $S(T)$ is equivalent to exponential integrability of $X(T)$. Inspecting the explicit relation for $X(s)$ in $(3.14)$, we see that $X(T)$ is exponentially integrable as long as

$$
\mathbb{E}_{Q}\left[\exp \left(\int_{0}^{T} \sigma(s) \mathrm{e}^{-\alpha(T-s)} d W_{1}(s)\right)\right]<\infty
$$


We claim that a sufficient condition for this to hold is that

$$
\int_{0}^{\infty}\left(\mathrm{e}^{0.5 \gamma^{*}\left(2 \alpha, \lambda_{j}\right) z}-1\right) \ell_{j}(d z)<\infty
$$

for $j=1, \ldots, n$, and $\gamma^{*}$ defined in (3.8). To show this, first note that by double conditioning using the $\sigma$-algebra $\mathcal{G}_{T}$ generated by $\sigma^{2}(s), s \leq T$, we find by independence between $B_{1}$ and $\sigma^{2}$ that

$$
\begin{aligned}
\mathbb{E}_{Q}\left[\exp \left(\int_{0}^{T} \sigma(s) \mathrm{e}^{-\alpha(T-s)} d W_{1}(s)\right)\right] & =\mathbb{E}_{Q}\left[\mathbb{E}_{Q}\left[\exp \left(\int_{0}^{T} \sigma(s) \mathrm{e}^{-\alpha(T-s)} d W_{1}(s)\right) \mid \mathcal{G}_{T}\right]\right] \\
& =\mathbb{E}\left[\exp \left(\frac{1}{2} \int_{0}^{T} \sigma^{2}(s) \mathrm{e}^{-2 \alpha(T-s)} d s\right)\right] .
\end{aligned}
$$

From $Y_{j}(s)$ in Lemma 3.1 we have

$$
\begin{aligned}
\int_{0}^{T} \sigma^{2}(s) \mathrm{e}^{-2 \alpha(T-s)} d s= & \sum_{j=1}^{n} w_{j} Y_{j}(0) \int_{0}^{T} \mathrm{e}^{-2 \alpha(T-s)} \mathrm{e}^{-\lambda_{j} s} d s \\
& \quad+\sum_{j=1}^{n} w_{j} \int_{0}^{T} \mathrm{e}^{-2 \alpha(T-s)} \int_{0}^{s} \mathrm{e}^{-\lambda_{j}(s-u)} d L_{j}(u) d s \\
= & \gamma\left(T ; 2 \alpha, \lambda_{j}\right)+\int_{0}^{T} \gamma\left(T-u ; 2 \alpha, \lambda_{j}\right) d L_{j}(u),
\end{aligned}
$$

after using the stochastic Fubini theorem. By independence of $L_{j}, j=1, \ldots, n$, we find that (3.15) holds whenever

$$
\mathbb{E}\left[\exp \left(\frac{1}{2} \int_{0}^{T} \gamma\left(T-u ; 2 \alpha, \lambda_{j}\right) d L_{j}(u)\right)\right]<\infty, j=1, \ldots, n .
$$

But $\gamma\left(u ; 2 \alpha, \lambda_{j}\right)$ is a non-negative function which has the maximum $\gamma^{*}\left(2 \alpha, \lambda_{j}\right)$, and $L_{j}$ being a subordinator implies the bound

$$
\int_{0}^{T} \gamma\left(T-u ; 2 \alpha, \lambda_{j}\right) d L_{j}(u) \leq \gamma^{*}\left(2 \alpha, \lambda_{j}\right) L_{j}(T)
$$

Finally, under condition (3.16) we have that

$$
\mathbb{E}\left[\exp \left(\frac{1}{2} \gamma^{*}\left(2 \alpha, \lambda_{j}\right) L_{j}(T)\right)\right]=\exp \left(T \int_{0}^{\infty}\left\{\mathrm{e}^{0.5 \gamma^{*}\left(2 \alpha, \lambda_{j}\right) z}-1\right\} \ell_{j}(d z)\right),
$$

from the definition of the moment generating function of a subordinator. This shows that (3.16) is a sufficient condition for the exponential integrability of $X(T)$ with respect to $Q$ for all $T \leq T_{\max }$. Note that it also is a sufficient condition for exponential integrability of $X(T)$ with respect to the market probability $P$.

In the next Proposition we state a semi-analytical expression for the forward price:

Proposition 3.6. Assume condition (3.16) holds. Then the forward price $f(t, T)$ at time $t \geq 0$ of a contract maturing at time $t \leq T \leq T_{\max }$ is

$$
f(t, T)=\Lambda_{f}(t, T) \Theta(T-t) S(t)^{\exp (-\alpha(T-t))} \exp (Z(t) \gamma(T-t ; \alpha, \beta)) H\left(t, T, Y_{1}(t), \ldots, Y_{n}(t)\right)
$$

where

$$
H\left(t, T, y_{1}, \ldots, y_{n}\right)=\mathbb{E}\left[\exp \left(\frac{1}{2} \int_{t}^{T}\left(\rho \eta \gamma(T-s ; \alpha, \beta)+\sigma(s) \mathrm{e}^{-\alpha(T-s)}\right)^{2} d s\right) \mid Y_{j}(t)=y_{j}\right]
$$

and

$$
\begin{aligned}
& \ln \Lambda_{f}(t, T)=\ln \Lambda(T)-\mathrm{e}^{-\alpha(T-t)} \ln \Lambda(t) \\
& \ln \Theta(u)=\left(\theta_{1}+\frac{\mu+\theta_{2}}{\beta}\right) \gamma(u ; \alpha, 0)-\frac{\mu+\theta_{2}}{\beta} \gamma(u ; \alpha, \beta)+\frac{1}{2} \eta^{2}\left(1-\rho^{2}\right) \int_{0}^{u} \gamma^{2}(s ; \alpha, \beta) d s .
\end{aligned}
$$


Proof. The forward price is

$$
f(t, T)=\mathbb{E}_{Q}\left[S(T) \mid \mathcal{F}_{t}\right]=\Lambda(T) \mathbb{E}_{Q}\left[\mathrm{e}^{X(T)} \mid \mathcal{F}_{t}\right] .
$$

Apply the explicit expression for the $Q$-dynamics of $X(T)$ given $X(t)$ and $Z(t)$ in (3.14), to obtain

$$
\begin{aligned}
& \mathbb{E}_{Q}\left[\mathrm{e}^{X(T)} \mid \mathcal{F}_{t}\right]= \exp \left(X(t) \mathrm{e}^{-\alpha(T-t)}+Z(t) \gamma(T-t ; \alpha, \beta)\right) \\
& \times \exp \left(\left(\theta_{1}+\frac{\mu+\theta_{2}}{\beta}\right) \gamma(T-t ; \alpha, 0)-\frac{\mu+\theta_{2}}{\beta} \gamma(T-t ; \alpha, \beta)\right) \\
& \times \mathbb{E}_{Q}\left[\exp \left(\int_{t}^{T}\left(\rho \eta \gamma(T-s ; \alpha, \beta)+\sigma(s) \mathrm{e}^{-\alpha(T-s)}\right) d W_{1}(s)\right) \mid \mathcal{F}_{t}\right] \\
& \times \mathbb{E}_{Q}\left[\exp \left(\eta \sqrt{1-\rho^{2}} \int_{t}^{T} \gamma(T-s ; \alpha, \beta) d V(s)\right) \mid \mathcal{F}_{t}\right] .
\end{aligned}
$$

Here, we have made use of the adaptedness of $X(t)$ and $Z(t)$ and the independence of $W_{1}$ and $V$. By the independent increment property of the Brownian motion $V$, we easily compute the second conditional expectation to be

$\mathbb{E}_{Q}\left[\exp \left(\eta \sqrt{1-\rho^{2}} \int_{t}^{T} \gamma(T-s ; \alpha, \beta) d V(s)\right) \mid \mathcal{F}_{t}\right]=\exp \left(\frac{1}{2} \eta^{2}\left(1-\rho^{2}\right) \int_{t}^{T} \gamma^{2}(T-s ; \alpha, \beta) d s\right)$.

Let now $\mathcal{G}_{t, T}$ be the product $\sigma$-algebra generated by the paths $\sigma(s) ; s \leq t \leq T$ and $\mathcal{F}_{t}$. By double condition, we have by independence of $\sigma(t)$ and $W_{1}(t)$ and the independent increment property of $W_{1}$,

$$
\begin{aligned}
\mathbb{E}_{Q}\left[\exp \left(\int_{t}^{T} \rho \eta \gamma(T-s ; \alpha, \beta)+\sigma(s) \mathrm{e}^{-\alpha(T-s)} d W_{1}(s)\right) \mid \mathcal{F}_{t}\right] \\
\quad=\mathbb{E}_{Q}\left[\mathbb{E}_{Q}\left[\exp \left(\int_{t}^{T} \rho \eta \gamma(T-s ; \alpha, \beta)+\sigma(s) \mathrm{e}^{-\alpha(T-s)} d W_{1}(s)\right) \mid \mathcal{G}_{t, T}\right] \mid \mathcal{F}_{t}\right] \\
\quad=\mathbb{E}\left[\exp \left(\frac{1}{2} \int_{t}^{T}\left(\rho \eta \gamma(T-s ; \alpha, \beta)+\sigma(s) \mathrm{e}^{-\alpha(T-s)}\right)^{2} d s\right) \mid \mathcal{F}_{t}\right] .
\end{aligned}
$$

By the Markov property of $Y_{j}(s)$ we find the function $H$. The proof is hence complete.

The forward price is explicit as a function of both the spot price and the seasonal level $Z(t)$. Moreover, it also varies explicitly as a function of the volatility of $S(t)$ through $H$ which depends on each of the volatility factors $Y_{1}(t), \ldots, Y_{n}(t)$. All these factor dependencies add up to a complex forward price evolution. In addition, we have two deterministic terms contributing to the shape of $f(t, T)$. First, the seasonal term $\Lambda_{f}(t, T)$, which can be interpreted as the change in seasonal level between today and maturity, adjusted by the mean-reversion between the two dates. The final term $\Theta(T-t)$ is coming from a drift contribution of $Z(t)$ as well as the market prices of risk $\theta_{1}$ and $\theta_{2}$. It is the only place where we see the effect of the pricing measure $Q$ explicitly in the forward price. The parameters $\theta_{1}$ and $\theta_{2}$ are unknown, since they cannot be estimated from observations of the spot price dynamics. The typical approach to estimate (or calibrate) the market prices of risk, is to minimize the distance between theoretical and observed forward prices. We remark that in practical situations, one would often prefer to use market prices of risk which are time dependent in order to facilitate for exact calibration to observed prices. At the expense of more technical computations and expressions, it is not a difficult task to extend our results to market prices of risk $\theta_{1}, \theta_{2}$ being functions of time.

In commodity markets, one is often interested in the risk premium, which is defined as the difference between the forward price and the predicted spot price:

$$
R_{Q}(t, T):=f(t, T)-\mathbb{E}\left[S(T) \mid \mathcal{F}_{t}\right] .
$$


Note that we indicate the dependency on the pricing measure $Q$ in the notation for the risk premium, which comes from the fact that we can express it as

$$
R_{Q}(t, T)=\mathbb{E}_{Q}\left[S(T) \mid \mathcal{F}_{t}\right]-\mathbb{E}\left[S(T) \mid \mathcal{F}_{t}\right] .
$$

We obtain the predicted spot price from Prop. 3.6 simply by choosing $\theta_{1}=\theta_{2}=0$. Hence, the risk premium becomes

$$
R_{Q}(t, T)=\Lambda_{f}(t, T) \widetilde{\Theta}_{0}(T-t) S(t)^{\exp (-\alpha(T-t))} \exp (Z(t) \gamma(T-t ; \alpha, \beta)) H\left(t, T ; Y_{1}(t), \ldots, Y_{n}(t)\right),
$$

with

$$
\begin{aligned}
\widetilde{\Theta}_{0}(u)= & \exp \left(\frac{\mu}{\beta}(\gamma(u ; \alpha, 0)-\gamma(u ; \alpha, \beta))+\frac{1}{2} \eta^{2}\left(1-\rho^{2}\right) \int_{0}^{u} \gamma^{2}(s ; \alpha, \beta) d s\right) \\
\times & \left\{\exp \left(\left(\theta_{1}+\frac{\theta_{2}}{\beta}\right) \gamma(u ; \alpha, 0)-\frac{\theta_{2}}{\beta} \gamma(u ; \alpha, \beta)\right)-1\right\} .
\end{aligned}
$$

Hence, we observe that all terms in the risk premium are positive, except possibly the expression inside the curly brackets of $\widetilde{\Theta}_{0}$. The sign of this term is determined by $\theta_{1}$ and $\theta_{2}$. In the simple case of $\theta_{2}=0$, we obtain a negative risk premium $R_{Q}$ if and only if $\theta_{1}<0$. A negative risk premium means that forward prices are lower than the predicted spot, which says that those selling the energy in the forward market accept a reduced price compared to what they predict to get if selling in the spot market instead. This can be a result of producers wishing to hedge their production using the forward market, and thereby accepting a discount in prices compared to the spot market. The risk premium can be intepreted as the insurance premium paid by those producers. A negative risk premium corresponds to a market in so-called backwardation.

The effect of $\theta_{2}$ on the risk premium is similar: suppose again for simplicity that $\theta_{1}=0$, and we see that the sign of the risk premium is negative whenever

$$
\theta_{2}(\gamma(u ; \alpha, 0)-\gamma(u ; \alpha, \beta))<0 .
$$

Define for the moment the function

$$
g(u)=\gamma(u ; \alpha, 0)-\gamma(u ; \alpha, \beta),
$$

for $u \geq 0$. Note that $g(0)=0$ and that $g(u)$ tends to $1 / \alpha>0$ when $u$ tends to infinity. Since $Z(t)$ is the mean-level of the spot price, it is natural to suppose that this mean-reverts slower than the actual spot price itself, yielding that $\alpha>\beta$ is a natural situation. We claim that $g(u)$ is strictly positive for $u>0$ in this case: indeed, the derivative of $g(u)$ is

$$
g^{\prime}(u)=\mathrm{e}^{-\alpha u}-\frac{1}{\alpha-\beta}\left(\beta \mathrm{e}^{-\beta u}-\alpha \mathrm{e}^{-\alpha u}\right) .
$$

But, $g^{\prime}(0)=2$ and $g^{\prime}(u)=0$ in only one point, namely

$$
u^{*}=\frac{\ln (2 \alpha-\beta)-\ln \beta}{\alpha-\beta} .
$$

Since $\alpha>\beta$, we have that $2 \alpha-\beta>\beta$, which implies that $u^{*}>0$. Hence, the continuous function $g(u)$ must be positive for $u>0$ since it starts increasing at $u=0$ from the origin, has only one extremal point at $u=u^{*}>0$ and is asymptotically converging to the positive constant $1 / \alpha$ at infinity. In conclusion, as long as $\alpha>\beta$, it holds that the risk premium is negative as long as $\theta_{2}<0$. We obtain the same result in the case $\alpha<\beta$, but with the additional condition $2 \alpha>\beta$, i.e., $\alpha<\beta<2 \alpha$.

We return to the analysis of the forward price $f(t, T)$. First, we present the dynamics of $F$ :

Proposition 3.7. Assume condition (3.16). The dynamics of $f(t, T)$ for $t \leq T$ is

$$
\begin{aligned}
\frac{d f(t, T)}{f(t-, T)}=\sigma(t) & \mathrm{e}^{-\alpha(T-t)} d W_{1}(t)+\eta \gamma(T-t ; \alpha, \beta) d W_{2}(t) \\
& +\sum_{j=1}^{n} \int_{0}^{\infty}\left\{\frac{H\left(t, T, Y_{1}(t-), \ldots, Y_{j}(t-)+z, \ldots, Y_{n}(t-)\right)}{H\left(t, T, Y_{1}(t-1), \ldots, Y_{n}(t-)\right)}-1\right\} \tilde{N}_{j}(d z, d t) .
\end{aligned}
$$


Proof. This follows from an application of Itô's Formula for jump processes exploiting the simplifying fact that $F$ is a $Q$-martingale.

We observe that the forward price evolves as a geometric jump-diffusion model, with the Brownian evolutions driven by the stochastic volatility $\sigma(t)$ discounted by the mean-reversion. In addition, we have explicit jump terms coming from the volatility. Although the spot price dynamics has continuous paths, the forward price dynamics will have jumps. Every time there is a change in volatility of the spot resulting from a jump in one or more of the subordinators $L_{j}$, the forward price will jump accordingly as well as getting an increase in volatility. In fact, the forward price will include a leverage effect in its price dynamics, since the volatility affects directly its price level. For the sake of illustration, consider the case of $n=1$, that is, only one factor $Y$ in the stochastic volatility specification. If $\rho \geq 0$ we find that the function

$$
y \mapsto\left(\rho \eta \gamma(T-s ; \alpha, \beta)+\sigma(s) \mathrm{e}^{-\alpha(T-s)}\right)^{2}
$$

is increasing, and therefore $y \mapsto H(t, T, y)$ must be increasing as well. Thus, the forward dynamics includes an inverse leverage effect in the sense that the forward price increases with the volatility $\sigma(t)$. The case $\rho<0$ is more involved, and here we potentially may have a "classical" leverage effect where forward prices are pulled down with higher volatility.

The price dynamics of the forward is semi-analytic in general, due to the function $H$ which is hard to compute explicitly. The problem is that the expectation in $H$ will involve a term

$$
\rho \eta \int_{t}^{T} \gamma(T-s ; \alpha, \beta) \mathrm{e}^{-\alpha(T-s)} \sigma(s) d s .
$$

We know $\sigma^{2}(s)$ explicitly, however, $\sigma(s)$ is the square root of a sum of OU-processes and the integral above seems hard to compute. In the case of no correlation between $B_{1}$ and $B_{2}$, that is, $\rho=0$, we can indeed obtain an explicit expression for $H$, and thus for the forward price. This is the content of the next Proposition:

Proposition 3.8. Assume condition (3.16), and suppose that $\rho=0$. Then

$$
H\left(t, T, y_{1}, \ldots, y_{n}\right)=\exp \left(\frac{1}{2} \sum_{j=1}^{n} w_{j} \gamma\left(T-t ; 2 \alpha, \lambda_{j}\right) y_{j}+\sum_{j=1}^{n} \int_{0}^{T-t} \psi_{j}\left(-\mathrm{i} \frac{w_{j}}{2} \gamma\left(s ; 2 \alpha, \lambda_{j}\right) d s\right)\right)
$$

Proof. For $\rho=0$, the function $H$ defined in Prop. 3.6 reduces to

$$
H\left(t, T, y_{1}, \ldots, y_{n}\right)=\mathbb{E}\left[\exp \left(\frac{1}{2} \int_{t}^{T} \sigma^{2}(s) \mathrm{e}^{-2 \alpha(T-s)} d s\right) \mid Y_{j}(t)=y_{j}\right] .
$$

From the explicit dynamics of $Y_{j}(s)$ given $Y_{j}(t)=y_{j}$ in Lemma 3.1, we find

$$
\begin{aligned}
\int_{t}^{T} \sigma^{2}(s) \mathrm{e}^{-2 \alpha(T-s)} d s= & \sum_{j=1}^{n} w_{j} y_{j} \int_{t}^{T} \mathrm{e}^{-\lambda_{j}(s-t)} \mathrm{e}^{-2 \alpha(T-s)} d s \\
& +\sum_{j=1}^{n} w_{j} \int_{t}^{T} \int_{t}^{s} \mathrm{e}^{-\lambda_{j}(s-u)} d L_{j}(u) \mathrm{e}^{-2 \alpha(T-s)} d s \\
= & \sum_{j=1}^{n} w_{j} y_{j} \gamma\left(T-t ; 2 \alpha, \lambda_{j}\right)+\sum_{j=1}^{n} w_{j} \int_{t}^{T} \int_{u}^{T} \mathrm{e}^{-\lambda_{j}(s-u)} \mathrm{e}^{-2 \alpha(T-s)} d s d L_{j}(u) \\
= & \sum_{j=1}^{n} w_{j} y_{j} \gamma\left(T-t ; 2 \alpha, \lambda_{j}\right)+\sum_{j=1}^{n} w_{j} \int_{t}^{T} \gamma\left(T-u ; 2 \alpha, \lambda_{j}\right) d L_{j}(u)
\end{aligned}
$$

where we have invoked the stochastic Fubini theorem in the second step. The Corollary follows by using the definition of the cumulant function of $L_{j}$ and condition (3.16). 
In the case of zero correlation between the driving Brownian motions, we also observe that the long-term influence of $H$ is simply a constant value,

$$
\lim _{T-t \rightarrow \infty} H\left(t, T, y_{1}, \ldots, y_{n}\right)=\exp \left(\sum_{j=1}^{n} \int_{0}^{\infty} \psi_{j}\left(-\mathrm{i} \frac{w_{j}}{2} \gamma\left(s ; 2 \alpha, \lambda_{j}\right) d s\right)\right),
$$

as long as the indefinite integrals exist.

Recall that the function $\gamma(u ; \alpha, \beta)$ starts at the origin for $u=0$, and tends to zero when $u \rightarrow \infty$. Moreover, it is non-negative and has a maximal value for $u^{*}=(\ln \alpha-\ln \beta) /(\alpha-\beta)>0$. Let now $n=1$ in Prop. 3.8 above. We see that the $H$ function is depending on the states as

$$
h(T-t)=\exp \left(\frac{1}{2} \gamma(T-t ; 2 \alpha, \lambda) y\right) .
$$

Hence, for $T-t=0$ we have $h(0)=1$, and when time-to-maturity $T-t$ tends to infinity, $h(T-t)$ tends to one. But, for

$$
T-t=\frac{\ln (2 \alpha)-\ln \lambda}{2 \alpha-\lambda}
$$

we have a a maximal value of $h$ strictly bigger than one. In fact, this maximum will be the product of the maximal value of $\gamma(u ; 2 \alpha, \lambda)$ and the state $y$. Thus, we find that $h(T-t)$ has a so-called hump-shaped structure, where the size of the hump will depend on the current state of the volatility, being $\sigma^{2}=y$. Thus, if we are in a market which currently is in a very volatile period, the model predicts a significant hump in the forward prices implied from the function $H(t, T, y)$. The hump will be in the shorter or longer end of the market, depending on the relative size between the speed of mean reversion $\alpha$ of the base component $X(t)$ and the speed of mean reversion of the volatility $\lambda$.

Note from the expression of the forward price in Prop. 3.6 that it is also explicitly a function of the current state of $Z(t)$, given by the term

$$
G(t, T)=\exp (Z(t) \gamma(T-t ; \alpha, \beta)) .
$$

As for the stochastic volatility, this term will also contribute with a hump shape, where the location and size of the hump will be dependent on the parameters $\alpha$ and $\beta$, and on the state of the stochastic mean level. If the mean level is very high, then the hump will be very pronounced, whereas a low mean level in the market will lead to a relatively small hump shape. Notice that for a given speed of mean reversion $\beta$ of the mean level process $Z(t)$, we find that the maximal value of $\gamma(u ; \alpha, \beta)$ will have the property that

$$
\lim _{\alpha \rightarrow 0} u^{*}=\lim _{\alpha \rightarrow 0} \frac{\ln \alpha-\ln \beta}{\alpha-\beta}=+\infty .
$$

Hence, the hump will be far out on the forward curve when the speed of mean reversion of the logarithmic price process is very slow. On the other hand, a big $\alpha$ relative to $\beta$ will give a hump in the very short end of the market, as

$$
\lim _{\alpha \rightarrow \infty} \frac{\ln \alpha-\ln \beta}{\alpha-\beta}=0 .
$$

We recall from (3.8) the maximal value of the function $\gamma(u ; \alpha, \beta)$ to be $\gamma^{*}(\alpha, \beta)$. Taking limits using L'Hopital's rule reveals that

$$
\lim _{\alpha \rightarrow 0} \gamma^{*}(\alpha, \beta)=\beta^{-1}
$$

while

$$
\lim _{\alpha \rightarrow \infty} \gamma^{*}(\alpha, \beta)=0
$$

Hence, a hump in the short end of the forward curve (implied by $\alpha$ being very big), is hardly visible except if the mean level is dramatically high. If the hump is far out (implied by a very slow mean reversion $\alpha$ ), we will see a hump basically given by $Z(t) / \beta$, which can become very large.

Remark that the terms $\Lambda_{f}(t, T)$ and $\Theta(T-t)$ in the forward price will scale the effects discussed above deterministically, as functions of the seasonality and market prices of risk. We might have 
humps arising from these terms as well, but such humps will occur at given times and of a given size. For example, a hump could occur every winter due to a seasonality effect in the market. The factor involving the current spot price $S(t)$ will yield a curve which decreases from the current spot in the short end to 1 in the long end (or the other way, if $S(t)<1$ ).

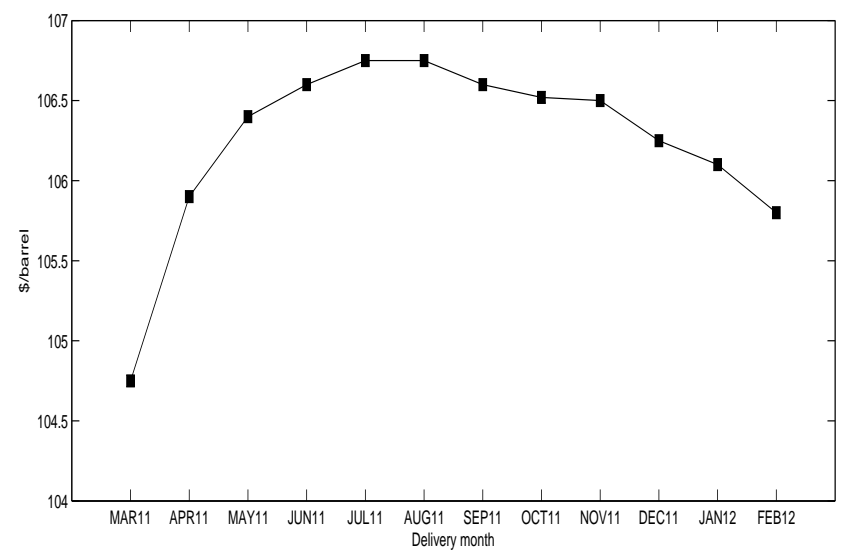

Figure 5. The WTI crude oil forward curve on February 282011

In Fig. 5 we have plotted the forward curve of WTI crude oil monthly contracts from February 28 2011. There is a clear hump shape in the forward curve, which may be attributed to an increase on the mean level of crude oil prices. In this period, the spot prices increased from about 90 Dollars per barrel to around 105, which may be attributed to an increase in the mean level $Z(t)$ (and possibly the volatility $\sigma(t)$ ). Our model predicts in such a case a hump shape, which therefore may explain the forward prices observed for WTI crude oil. (see Geman [17] for a dicussion of hump shaped forward curves for Brent oil).

We move on with our analysis of the forward prices with an investigation of the effect of the correlation $\rho$. In the case when $\rho \leq 0$, we have the trivial majorization

$$
\left(\sigma(s) \mathrm{e}^{-\alpha(T-s)}+\rho \eta \gamma(T-s ; \alpha, \beta)\right)^{2} \leq \sigma^{2}(s) \mathrm{e}^{-2 \alpha(T-s)}+\rho^{2} \eta^{2} \gamma^{2}(T-s ; \alpha, \beta) .
$$

From Props. 3.6-3.8, it follows that

$$
f(t, T) \leq f_{0}(t, T),
$$

where $f_{0}$ denotes the forward price for $\rho=0$. A negative correlation will lead to more concentrated spot prices compared to no or positive correlation. Less variation in spot price reduces the forward price. From the same arguments, the opposite holds when $\rho \geq 0$, that is,

$$
f(t, T) \geq f_{0}(t, T) .
$$

A positive correlation creates a bigger variation in the spot prices, and we recognize the effect as higher forward prices compared to the benchmark at zero correlation.

For a negative correlation $\rho$, we have that the function $H\left(t, T, y_{1}, \ldots, y_{n}\right)$ is bounded by the expression given in Prop. 3.8 (being the function $H$ with $\rho=0$ ). This bound has a stationary limit under some mild hypothesis on the cumulant functions of the subordinators driving the volatility. Hence, as time to maturity goes to infinity, we find that the function $H$ will be contained within the interval $(1, c)$, where $c$ is the stationary limit of $H$ for $\rho=0$.

Let us consider the case with positive correlation $\rho>0$. The lower limit for $H$ will be $c$, the limit of $H$ in the zero correlation case. However, we can also bound $H$ from above. By elementary inequalities, it holds that

$$
\frac{1}{2}\left(\rho \eta \gamma(T-s ; \alpha, \beta)+\sigma(s) \mathrm{e}^{-\alpha(T-s)}\right)^{2} \leq \rho^{2} \eta^{2} \gamma^{2}(T-s ; \alpha, \beta)+\sigma^{2}(s) \mathrm{e}^{-2 \alpha(T-s)} .
$$


Thus,

$$
\begin{aligned}
H\left(t, T, y_{1}, \ldots, y_{n}\right) \leq & \exp \left(\rho^{2} \eta^{2} \int_{0}^{T-t} \gamma^{2}(s ; \alpha, \beta) d s\right) \\
& \times \mathbb{E}\left[\exp \left(\int_{t}^{T} \sigma^{2}(s) \mathrm{e}^{-2 \alpha(T-s)} d s\right) \mid Y_{j}(t)=y_{j}\right] .
\end{aligned}
$$

As $\gamma(s ; \alpha, \beta)$ is the difference of two exponentially decaying functions, the first term above has a limit. Appealing to the same arguments as in the proof of Prop. 3.8 reveals that the expectation operator also has a limit when $T-t$ tend to infinity. Hence, $H$ is bounded from above when $T-t$ becomes large, and there will be an interval $(c, d)$ within which $H$ is contained. We leave the analysis of the asymptotic limit of $H$ when $T-t$ tends to infinity as an open question.

We end this Section with an example of a stochastic volatility specification. Let $n=1$, such that $\sigma^{2}(t)=Y(t)$, and assume that the subordinator $L$ driving $Y$ is a compound Poisson process with exponentially distributed jumps, that is,

$$
L(t)=\sum_{k=1}^{N(t)} J_{k}
$$

where $N(t)$ is a Poisson process with intensity $\delta>0$ and $\left\{J_{k}\right\}$, are independent and distributed according to an exponential distribution with mean $1 / a, a>0$. We first compute the cumulant of $J$ :

$$
\psi_{J}(x)=\ln \mathbb{E}[\exp (\mathrm{i} x J)]=\ln \int_{0}^{\infty} \mathrm{e}^{\mathrm{i} x y} a \mathrm{e}^{-a y} d y=\ln \left(\frac{a}{a-\mathrm{i} x}\right) .
$$

We observe that the moment generating function $\phi_{J}(y)=\psi_{J}(-\mathrm{i} y)$ exists for all $y \leq a$. By conditioning, we can next compute the cumulant of $L(1)$ :

$$
\begin{aligned}
\psi(x) & =\ln \mathbb{E}[\exp (\mathrm{i} x L(1)] \\
& =\mathbb{E}\left[\exp \left(\mathrm{i} x \sum_{k=1}^{N(1)} J_{k}\right)\right] \\
& =\ln \mathrm{e}^{-\delta} \sum_{n=0}^{\infty} \frac{\delta^{n}}{n !} \mathbb{E}[\exp (\mathrm{i} x J)]^{n} \\
& =\delta\left(\mathrm{e}^{\psi_{J}(x)}-1\right) \\
& =\delta \frac{\mathrm{i} x}{a-\mathrm{i} x} .
\end{aligned}
$$

Denoting by $\psi_{\sigma^{2}(s)}(t, x)$ the conditional cumulant of $\sigma^{2}(s)$ given $\mathcal{F}_{t}, s \geq t$, we find by a direct computation that

$$
\begin{aligned}
\psi_{\sigma^{2}(s)}(t, x) & =\ln \mathbb{E}\left[\exp \left(\mathrm{i} x \sigma^{2}(s)\right) \mid \mathcal{F}_{t}\right] \\
& =\ln \mathbb{E}\left[\exp \left(\mathrm{i} x \sigma^{2}(t) \mathrm{e}^{-\lambda(s-t)}+\int_{t}^{s} \mathrm{e}^{-\lambda(s-u)} d L(u)\right) \mid \mathcal{F}_{t}\right] \\
& =\mathrm{i} x \sigma^{2}(t) \mathrm{e}^{-\lambda(s-t)}+\ln \mathbb{E}\left[\exp \left(\mathrm{i} x \int_{t}^{s} \mathrm{e}^{-\lambda(s-u)} d L(u)\right)\right] \\
& =\mathrm{i} x \sigma^{2}(t) \mathrm{e}^{-\lambda(s-t)}+\int_{0}^{s-t} \psi\left(x \mathrm{e}^{-\lambda u}\right) d u \\
& =\mathrm{i} x \sigma^{2}(t) \mathrm{e}^{-\lambda(s-t)}+\delta \int_{0}^{s-t} \frac{\mathrm{i} x \mathrm{e}^{-\lambda u}}{a-\mathrm{i} x \mathrm{e}^{-\lambda u} d u} \\
& =\mathrm{i} x \sigma^{2}(t) \mathrm{e}^{-\lambda(s-t)}+\frac{\delta}{\lambda} \ln \left(\frac{a-\mathrm{i} x \mathrm{e}^{-\lambda(s-t)}}{a-\mathrm{i} x}\right) .
\end{aligned}
$$


Here we have used the $\mathcal{F}_{t}$-measurability of $\sigma^{2}(t)$ and the independent increment property of $L$. As $s-t \rightarrow \infty$, we find that

$$
\lim _{s-t \rightarrow \infty} \psi_{\sigma^{2}(s)}(t, x)=\ln \left(1-\mathrm{i} \frac{x}{a}\right)^{-\delta / \lambda} .
$$

Hence, in stationarity $\sigma^{2}(s)$ becomes $\Gamma$ distributed with shape parameter $\delta / \lambda$ and scale $1 / a$. The probability density function of this distribution is given as

$$
p_{\Gamma}(x ; k, a)=\frac{a^{k}}{\Gamma(k)} x^{k-1} \mathrm{e}^{-a x},
$$

with $k=\delta / \lambda$. is

Let us analyse $\sigma(t)$, the volatility, in this case. We find that the characteristic function of $\sigma(t)$

$$
\begin{aligned}
\mathbb{E}\left[\mathrm{e}^{\mathrm{i} x \sigma(t)}\right] & =\mathbb{E}\left[\mathrm{e}^{\mathrm{i} x \sqrt{\sigma^{2}(t)}}\right] \\
& =\int_{0}^{\infty} \mathrm{e}^{\mathrm{i} x \sqrt{y}} P_{\sigma^{2}(t)}(d y),
\end{aligned}
$$

with $P_{\sigma^{2}(t)}$ being the distribution function of $\sigma^{2}(t)$. However, as we know from above, $P_{\sigma^{2}(t)}(d y) \rightarrow$ $p_{\Gamma}(y ; k, a) d y$ as $t \rightarrow \infty$. Thus,

$$
\lim _{t \rightarrow \infty} \mathbb{E}\left[\mathrm{e}^{\mathrm{i} x \sigma(t)}\right]=\int_{0}^{\infty} \mathrm{e}^{\mathrm{i} x \sqrt{y}} p_{\Gamma}(y ; k, a) d y .
$$

As this integral can be computed (yielding a very long expression consisting of Whittaker parabolic and trigonometric functions), we find an expression for the characteristic function of the stationary distribution of $\sigma(t)$. The mean value of the stationary distribution is, however, expressible in a rather compact form (using Maple):

$$
\lim _{t \rightarrow \infty} \mathbb{E}[\sigma(t)]=-\frac{2 \pi \sec (k \pi)}{(2 k+1) \sqrt{a} \Gamma(k) \Gamma\left(-k-\frac{1}{2}\right)} .
$$

The stationary mean of the volatility is therefore proportional to the square-root of the mean jump size $1 / a$ of $L$. For example, if $\delta=\lambda$ (implying that $k=1$ ), we find

$$
\lim _{t \rightarrow \infty} \mathbb{E}[\sigma(t)]=\frac{\sqrt{\pi}}{2 \sqrt{a}} .
$$

Recall the analysis of the autocorrelation structure of $X(t)$ leading to (3.10), where the stationary mean value of the volatility is appearing explicitly.

\section{Cross-COmmodity DeRIVAtives}

In this Section we focus on cross-commodity models of energy prices. We want to investigate pricing of simple spread options in a cross-commodity multi-factor model, as well as sensitivity measures and dependency risk.

4.1. A Margrabe Formula for Energy markets. We want to derive a Margrabe formula for energy markets. In the energy markets, there exist a plethora of various spread options, and we focus on exchange-type options on spot, including spark and dark spreads. We recall that a stationary model is the natural dynamics for energy spot prices rather than geometric Brownian motions, calling for an extension of the classical Margrabe formula (see Margrabe [23]). Moreover, spikes call for non-Gaussian models, which further complicates the pricing of spread options for energy markets.

Letting $S_{1}(t)$ and $S_{2}(t)$ be the spot price dynamics of two energies, we are interested in deriving a price for an option on the spread between them, that is,

$$
P(t)=\mathrm{e}^{-r(T-t)} \mathbb{E}\left[\left(S_{1}(T)-h S_{2}(T)\right)^{+} \mid \mathcal{F}_{t}\right]
$$

where $h>0$ is a constant, $(x)^{+}=\max (x, 0)$ and $r>0$ a constant risk-free interest rate. For simplicity, we suppose throughout this Section that the pricing measure $Q$ is chosen to be the 
market probability $P, Q=P$, i.e., there is no market price of risk. We may view the situation alternatively as the spot being defined under the pricing measure directly, interpreting $P$ as this one. Obviously, we assume that $S_{1}$ and $S_{2}$ are integrable in order to make the expectation welldefined.

In the classical cases of energy spreads, $S_{1}$ may be the price of electricity, and $S_{2}(t)$ the fuel. For example, we can have that $S_{2}$ is the price of gas, and in that case $h$ is known as the heat rate, the factor converting the price of gas energy into the electricity equivalent.

Suppose that the price dynamics of $S_{i}, i=1,2$ are defined as

$$
S_{i}(t)=\Lambda_{i}(t) \exp \left(X_{i}(t)+Y_{i}(t)\right)
$$

where

$$
\begin{aligned}
d X_{i}(t) & =\left(\mu_{i}-\alpha_{i} X_{i}(t)\right) d t+\sigma_{i} d B_{i}(t) \\
d Y_{i}(t) & =\left(\gamma_{i}-\beta_{i} Y_{i}(t)\right) d t+d L_{i}(t) .
\end{aligned}
$$

Here, $L=\left(L_{1}, L_{2}\right)$ is a bivariate square-integrable Lévy process independent of $B_{1}, B_{2}$, which are two correlated Brownian motions with correlation coefficient $\rho$. Furthermore, $\mu_{i}, \alpha_{i}, \gamma_{i}, \beta_{i}$ and $\sigma_{i}$, for $i=1,2$ are all constants, with $\alpha_{i}, \beta_{i}$ and $\sigma_{i}$ assumed positive. In the next Lemma, we state the explicit dynamics of the OU-processes:

Lemma 4.1. For $0 \leq t \leq s$, it holds that

$$
X_{i}(s)=X_{i}(t) \mathrm{e}^{-\alpha_{i}(s-t)}+\frac{\mu_{i}}{\alpha_{i}}\left(1-\mathrm{e}^{-\alpha_{i}(s-t)}\right)+\int_{t}^{s} \sigma_{i} \mathrm{e}^{-\alpha_{i}(s-u)} d B_{i}(u),
$$

and

for $i=1,2$.

$$
Y_{i}(s)=Y_{i}(t) \mathrm{e}^{-\beta_{i}(s-t)}+\frac{\gamma_{i}}{\beta_{i}}\left(1-\mathrm{e}^{-\beta_{i}(s-t)}\right)+\int_{t}^{s} \mathrm{e}^{-\beta_{i}(s-u)} d L_{i}(u)
$$

Proof. This is a straightforward application of Itô's Formula for jump processes.

In order for the spread option price to be well-defined, we need that $\left(S_{1}(T)-S_{2}(T)\right)^{+}$has finite expectation, which is true if both $S_{1}(T)$ and $S_{2}(T)$ have finite expectation. A sufficient condition for this to hold is that $\int_{0}^{t} \exp \left(-\beta_{i}(t-s)\right) d L_{i}(s), i=1,2$ have finite exponential moment. To this end, introduce the rectangle $R \subset \mathbb{R}^{2}$ including the origin defined as all pairs $(a, b)$ such that

$$
\int_{\mathbb{R}_{0}^{2}}\left\{\mathrm{e}^{a x+b y}-1\right\} \ell(d x, d y)<\infty
$$

with $\ell$ being the Lévy measure of $L$. A sufficient condition for $\int_{0}^{t} \exp \left(-\beta_{i}(t-s)\right) d L_{i}(s), i=1,2$ to have finite exponential moments is that $R=[0,1]^{2}$ in (4.5). We assume this is true from now on.

Hence, by appealing to $\mathcal{F}_{t}$-adaptedness, we find that the conditional expectation for the price can be expressed as

$$
\begin{aligned}
\mathbb{E}[ & \left.\left(S_{1}(T)-h S_{2}(T)\right)^{+} \mid \mathcal{F}_{t}\right] \\
= & \mathbb{E}\left[\left(\Lambda_{1}(T) \mathrm{e}^{X_{1}(T)+Y_{1}(T)}-h \Lambda_{2}(T) \mathrm{e}^{X_{2}(T)+Y_{2}(T)}\right)^{+} \mid \mathcal{F}_{t}\right] \\
= & C_{1}\left(t, T, X_{1}(t), Y_{1}(t)\right) \\
& \times \mathbb{E}\left[\mathrm{e}^{\Xi_{2}(t, T)+\Psi_{2}(t, T)}\left(\mathrm{e}^{\Xi_{1}(t, T)-\Xi_{2}(t, T)+\Psi_{1}(t, T)-\Psi_{2}(t, T)}-h \frac{C_{2}\left(t, T, X_{2}(t), Y_{2}(t)\right)}{C_{1}\left(t, T, X_{1}(t), Y_{1}(t)\right)}\right)^{+} \mid \mathcal{F}_{t}\right]
\end{aligned}
$$

where

(4.6)

$$
C_{i}(t, T, x, y)=\Lambda_{i}(T) \exp \left(\frac{\mu_{i}}{\alpha_{i}}\left(1-\mathrm{e}^{-\alpha_{i}(T-t)}\right)+\frac{\gamma_{i}}{\beta_{i}}\left(1-\mathrm{e}^{-\beta_{i}(T-t)}\right)+x \mathrm{e}^{-\alpha_{i}(T-t)}+y \mathrm{e}^{-\beta_{i}(T-t)}\right),
$$


and

$$
\begin{aligned}
& \Xi_{i}(t, T)=\int_{t}^{T} \sigma_{i} \mathrm{e}^{-\alpha_{i}(T-u)} d B_{i}(u) \\
& \Psi_{i}(t, T)=\int_{t}^{T} \mathrm{e}^{-\beta_{i}(T-u)} d L_{i}(u),
\end{aligned}
$$

for $i=1,2$. Hence, due to $\mathcal{F}_{t}$-adaptedness and independent increment property of the Brownian motions and Lévy processes, the pricing $P(t)$ of the spread option entails in computing the expectation

$$
p(t, T, K)=\mathbb{E}\left[\mathrm{e}^{\Xi_{2}(t, T)+\Psi_{2}(t, T)}\left(\mathrm{e}^{\Xi_{1}(t, T)-\Xi_{2}(t, T)+\Psi_{1}(t, T)-\Psi_{2}(t, T)}-K\right)^{+}\right],
$$

for a deterministic strike price $K$ depending on the current states of the factors in the spot prices as well as current time $t$ and maturity $T$. We write $K$ in the sequel for simplicity.

In the next Proposition, we compute $p(t, T, K)$ in (4.9) using the change of measure technique with respect to Brownian motion (see Carmona and Durrleman [14] for this idea, used to derive the classical Margrabe formula):

Proposition 4.2. The price $p(t, T, K)$ defined in (4.9) is given by

$$
p(t, T, K)=\exp \left(\frac{\sigma_{2}^{2}}{4 \alpha_{2}}\left(1-\mathrm{e}^{-2 \alpha_{2}(T-t)}\right)\right) \mathbb{E}\left[\mathrm{e}^{\Psi_{2}(t, T)} F\left(a(t, T), \Sigma(t, T), K ; \Psi_{1}(t, T)-\Psi_{2}(t, T)\right)\right]
$$

where $F(a, b, K ; x)$ is defined as

$$
F(a, b, K ; x)=\exp \left(a+x+\frac{1}{2} b^{2}\right) N\left(b+\frac{a+x-\ln K}{b}\right)-K N\left(\frac{a+x-\ln K}{b}\right),
$$

with $N$ being the cumulative standard normal distribution. Furthemore,

and variance

$$
a(t, T)=\rho \frac{\sigma_{1} \sigma_{2}}{\alpha_{1}+\alpha_{2}}\left(1-\mathrm{e}^{-\left(\alpha_{1}+\alpha_{2}\right)(T-t)}\right)-\frac{\sigma_{2}^{2}}{2 \alpha_{2}}\left(1-\mathrm{e}^{-2 \alpha_{2}(T-t)}\right),
$$

$$
\Sigma^{2}(t, T)=\frac{\sigma_{1}^{2}}{2 \alpha_{1}}\left(1-\mathrm{e}^{-2 \alpha_{1}(T-t)}\right)-2 \rho \frac{\sigma_{1} \sigma_{2}}{\alpha_{1}+\alpha_{2}}\left(1-\mathrm{e}^{-\left(\alpha_{1}+\alpha_{2}\right)(T-t)}\right)+\frac{\sigma_{2}^{2}}{2 \alpha_{2}}\left(1-\mathrm{e}^{-2 \alpha_{2}(T-t)}\right) .
$$

Proof. Recall that $\left(\Psi_{1}, \Psi_{2}\right)$ and $\left(\Xi_{1}, \Xi_{2}\right)$ are independent, and hence by the tower property of conditional expectation we find

$$
\begin{aligned}
\mathbb{E} & {\left[\mathrm{e}^{\Xi_{2}(t, T)+\Psi_{2}(t, T)}\left(\mathrm{e}^{\Xi_{1}(t, T)-\Xi_{2}(t, T)+\Psi_{1}(t, T)-\Psi_{2}(t, T)}-K\right)^{+}\right] } \\
& =\mathbb{E}\left[\mathrm{e}^{\Psi_{2}(t, T)} \mathbb{E}\left[\mathrm{e}^{\Xi_{2}(t, T)}\left(\mathrm{e}^{\Xi_{1}(t, T)-\Xi_{2}(t, T)+\Psi_{1}(t, T)-\Psi_{2}(t, T)}-K\right)^{+} \mid \Psi_{1}(t, T), \Psi_{2}(t, T)\right]\right] .
\end{aligned}
$$

Thus, our first problem is to compute the inner conditional expectation, which amounts to calculating the expectation

for a constant $m=\Psi_{1}(t, T)-\Psi_{2}(t, T)$.

$$
\mathbb{E}\left[\mathrm{e}^{\Xi_{2}(t, T)}\left(\mathrm{e}^{\Xi_{1}(t, T)-\Xi_{2}(t, T)+m}-K\right)^{+}\right],
$$

To this end, introduce the martingale process $Z(s)$ on $t \leq s \leq T$ as

$$
Z(s)=\exp \left(\int_{t}^{s} \sigma_{2} \mathrm{e}^{-\alpha_{2}(T-u)} d B_{2}(u)-\frac{\sigma_{2}^{2}}{4 \alpha_{2}}\left(\mathrm{e}^{-2 \alpha_{2}(T-s)}-\mathrm{e}^{-2 \alpha_{2}(T-t)}\right)\right),
$$

which, by Girsanov's Theorem is the density process of an equivalent probability $P^{*}$ and such that

$$
d W(s)=d B_{2}(s)-\sigma_{2} \mathrm{e}^{-\alpha_{2}(T-s)} d s,
$$

is a $P^{*}$-Brownian motion on $s \in[t, T]$. Thus,

$\mathbb{E}\left[\mathrm{e}^{\Xi_{2}(t, T)}\left(\mathrm{e}^{\Xi_{1}(t, T)-\Xi_{2}(t, T)+m}-K\right)^{+}\right]=\mathrm{e}^{\frac{\sigma_{2}^{2}}{4 \alpha_{2}}\left(1-\mathrm{e}^{-2 \alpha_{2}(T-t)}\right)} \mathbb{E}\left[Z(T)\left(\left(\mathrm{e}^{\Xi_{1}(t, T)-\Xi_{2}(t, T)+m}-K\right)^{+}\right]\right.$ 


$$
=\mathrm{e}^{\frac{\sigma_{2}^{2}}{4 \alpha_{2}}\left(1-\mathrm{e}^{-2 \alpha_{2}(T-t)}\right)} \mathbb{E}_{*}\left[\left(\mathrm{e}^{\Xi_{1}(t, T)-\Xi_{2}(t, T)+m}-K\right)^{+}\right],
$$

where $\mathbb{E}_{*}$ is the expectation operator under $P^{*}$. Since $B_{1}$ and $B_{2}$ are correlated Brownian motions, we find, for an independent Brownian motion $B$, that

$$
B_{1}(t)=\rho B_{2}(t)+\sqrt{1-\rho^{2}} B(t) .
$$

Hence,

$$
\begin{aligned}
\Xi_{1}(t, T)-\Xi_{2}(t, T) & =\int_{t}^{T} \sigma_{1} \mathrm{e}^{-\alpha_{1}(T-u)} d B_{1}(u)-\int_{t}^{T} \sigma_{2} \mathrm{e}^{-\alpha_{2}(T-u)} d B_{2}(u) \\
& =\int_{t}^{T} \rho \sigma_{1} \mathrm{e}^{-\alpha_{1}(T-u)}-\sigma_{2} \mathrm{e}^{-\alpha_{2}(T-u)} d B_{2}(u)+\int_{t}^{T} \sqrt{1-\rho^{2}} \sigma_{1} \mathrm{e}^{-\alpha_{1}(T-u)} d B(u) \\
& =\int_{t}^{T} \rho \sigma_{1} \mathrm{e}^{-\alpha_{1}(T-u)}-\sigma_{2} \mathrm{e}^{-\alpha_{2}(T-u)} d W(u)+\int_{t}^{T} \sqrt{1-\rho^{2}} \sigma_{1} \mathrm{e}^{-\alpha_{1}(T-u)} d B(u) \\
& \quad+\int_{t}^{T} \sigma_{2} \mathrm{e}^{-\alpha_{2}(T-u)}\left(\rho \sigma_{1} \mathrm{e}^{-\alpha_{1}(T-u)}-\sigma_{2} \mathrm{e}^{-\alpha_{2}(T-u)}\right) d u
\end{aligned}
$$

Note that $B$ is a Brownian motion under $P^{*}$, since it is independent of $B_{2}$. Thus, under $P^{*}$, we have that $\Xi_{1}(t, T)-\Xi_{2}(t, T)+m$ is a normally distributed random variable, with mean equal to $m+a(t, T)$ where

$$
\begin{aligned}
a(t, T) & =\int_{t}^{T} \sigma_{2} \mathrm{e}^{-\alpha_{2}(T-u)}\left(\rho \sigma_{1} \mathrm{e}^{-\alpha_{1}(T-u)}-\sigma_{2} \mathrm{e}^{-\alpha_{2}(T-u)}\right) d u \\
& =\rho \frac{\sigma_{1} \sigma_{2}}{\alpha_{1}+\alpha_{2}}\left(1-\mathrm{e}^{-\left(\alpha_{1}+\alpha_{2}\right)(T-t)}\right)-\frac{\sigma_{2}^{2}}{2 \alpha_{2}}\left(1-\mathrm{e}^{-2 \alpha_{2}(T-t)}\right),
\end{aligned}
$$

and variance

$$
\begin{aligned}
\Sigma^{2}(t, T) & =\int_{t}^{T}\left(\rho \sigma_{1} \mathrm{e}^{-\alpha_{1}(T-u)}-\sigma_{2} \mathrm{e}^{-\alpha_{2}(T-u)}\right)^{2}+\left(1-\rho^{2}\right) \sigma_{1}^{2} \mathrm{e}^{-2 \alpha_{1}(T-u)} d u \\
& =\frac{\sigma_{1}^{2}}{2 \alpha_{1}}\left(1-\mathrm{e}^{-2 \alpha_{1}(T-t)}\right)-2 \rho \frac{\sigma_{1} \sigma_{2}}{\alpha_{1}+\alpha_{2}}\left(1-\mathrm{e}^{-\left(\alpha_{1}+\alpha_{2}\right)(T-t)}\right)+\frac{\sigma_{2}^{2}}{2 \alpha_{2}}\left(1-\mathrm{e}^{-2 \alpha_{2}(T-t)}\right) .
\end{aligned}
$$

Using the same line of derivations as for a call option price, we find that

$$
\begin{gathered}
\mathbb{E}_{*}\left[\left(\mathrm{e}^{\Xi_{1}(t, T)-\Xi_{2}(t, T)+m}-K\right)^{+}\right]=\mathrm{e}^{a(t, T)+m+\frac{1}{2} \Sigma^{2}(t, T)} N\left(\Sigma(t, T)+\frac{a(t, T)+m-\ln K}{\Sigma(t, T)}\right) \\
-K N\left(\frac{a(t, T)+m-\ln K}{\Sigma(t, T)}\right) .
\end{gathered}
$$

Thus, by appealing to the definition of $F$, the proof is complete.

In the Proposition above, we have reduced the problem of finding $p(t, T, K)$ to computing an expectation of a function of the difference of two Lévy integrals. Thus, we face the problem of pricing a spread option again, but now reduced to being a spread between the jump terms only. As it turns out, one may again appeal to a change of measure to express this expectation. Moreover, it is advantagous to apply the Fourier transform to obtain a "closed-form" expression for $p(t, T, K)$ that is possible to compute numerically by fast Fourier transform methods.

To prepare for this, we make a small excursion into the Esscher transform for bivariate Lévy processes. On $t \in[0, T]$, define the stochastic process

$$
Z(t)=\exp \left(\int_{0}^{t} \theta_{1}(s) d L_{1}(s)+\int_{0}^{t} \theta_{2}(s) d L_{2}(s)-\int_{0}^{t} \phi\left(\theta_{1}(s), \theta_{2}(s)\right) d s\right),
$$

with $\theta_{1}, \theta_{2}$ being two bounded measurable functions, and $\phi(x, y)$ the log-moment generating function of $L=\left(L_{1}, L_{2}\right)$. Note that by our exponential integrability assumption on $L, \phi(x, y)$ is 
well-defined, and it follows that $Z(t)$ is a martingale process on $[0, T]$. We introduce the probability measure $\widetilde{P}$ with density

$$
\left.\frac{d \widetilde{P}}{d P}\right|_{\mathcal{F}_{t}}=Z(t), t \leq T .
$$

This is known as the Esscher transform of $L$. Define the conditional cumulant function of $L$ under $\widetilde{P}$ as

$$
\psi_{\widetilde{P}}(s, t, x, y):=\ln \mathbb{E}_{\widetilde{P}}\left[\exp \left(\mathrm{i} x\left(L_{1}(t)-L_{1}(s)\right)+\mathrm{i} y\left(L_{2}(t)-L_{2}(s)\right)\right) \mid \mathcal{F}_{s}\right]
$$

for $T \geq t \geq s \geq 0$. It turns out that $L$ becomes a bivariate independent increment process (see Benth et al. [4]) under $\widetilde{P}$, with characteristics given in the next Lemma:

Lemma 4.3. Under $\widetilde{P}, L$ has a conditional cumulant function

$$
\psi_{\widetilde{P}}(s, t, x, y)=\int_{s}^{t} \psi\left(x-\mathrm{i} \theta_{1}(u), y-\mathrm{i} \theta_{2}(u)\right) d u-\int_{s}^{t} \psi\left(-\mathrm{i} \theta_{1}(u),-\mathrm{i} \theta_{2}(u)\right) d u,
$$

where $\psi(x, y)$ is the cumulant of $L$ under $P$.

Proof. With $T \geq t \geq s \geq 0$, the conditional characteristic function becomes, by using Bayes' Formula and the independent increment property of Lévy processes,

$$
\begin{aligned}
\mathbb{E}_{\widetilde{P}} & {\left[\exp \left(\mathrm{i} x\left(L_{1}(t)-L_{1}(s)\right)+\mathrm{i} y\left(L_{2}(t)-L_{2}(s)\right)\right) \mid \mathcal{F}_{s}\right] } \\
& =\mathbb{E}\left[\frac{Z(t)}{Z(s)} \exp \left(\mathrm{i} x\left(L_{1}(t)-L_{1}(s)\right)+\mathrm{i} y\left(L_{2}(t)-L_{2}(s)\right)\right) \mid \mathcal{F}_{s}\right] \\
& =\exp \left(-\int_{s}^{t} \phi\left(\theta_{1}(u), \theta_{2}(u)\right) d u\right) \mathbb{E}\left[\exp \left(\int_{s}^{t} \mathrm{i} x+\theta_{1}(s) d L_{1}(s)+\int_{s}^{t} \mathrm{i} y+\theta_{2}(u) d L_{2}(u)\right) \mid \mathcal{F}_{s}\right] \\
& =\exp \left(-\int_{s}^{t} \phi\left(\theta_{1}(u), \theta_{2}(u)\right) d u\right) \mathbb{E}\left[\exp \left(\int_{s}^{t} \mathrm{i} x+\theta_{1}(s) d L_{1}(s)+\int_{s}^{t} \mathrm{i} y+\theta_{2}(u) d L_{2}(u)\right)\right] \\
& =\exp \left(\int_{s}^{t} \psi\left(x-\mathrm{i} \theta_{1}(u), y-\mathrm{i} \theta_{2}(u)\right) d u-\int_{s}^{t} \psi\left(-\mathrm{i} \theta_{1}(u),-\mathrm{i} \theta_{2}(u)\right) d u\right)
\end{aligned}
$$

Hence, the proof is complete.

By the Lévy-Kintchine formula for $L$, we have that

$$
\begin{aligned}
\psi_{\widetilde{P}}(s, t, x, y)=\mathrm{i} & \left\langle\int_{t}^{s}\left(\xi+\int_{|z|<1} z\left\{\mathrm{e}^{\langle z, \theta(u)\rangle}-1\right\} \ell(d z)\right) d u, w\right\rangle \\
& +\int_{t}^{s} \int_{\mathbb{R}^{2}}\left\{\mathrm{e}^{\mathrm{i}\langle z, w\rangle}-1-\mathrm{i}\langle z, w\rangle 1_{|z|<1}\right\} \mathrm{e}^{\langle z, \theta(u)\rangle} \ell(d z) d u,
\end{aligned}
$$

where $w:=(x, y), \theta(u)=\left(\theta_{1}(u), \theta_{2}(u)\right), \xi \in \mathbb{R}^{2}$ is the drift of $L$ and $\ell(d z)$ is the Lévy measure of $L$ defined on $\mathbb{R}^{2} \backslash\{0\}$. The drift will change from $\xi$ to

$$
\int_{t}^{s}\left(\xi+\int_{|z|<1} z\left\{\mathrm{e}^{\langle z, \theta(u)\rangle}-1\right\} \ell(d z)\right) d u,
$$

under $\widetilde{P}$. Moreover, $L$ will have a time-dependent jump measure under $\widetilde{P}$ given by

$$
\ell_{\widetilde{P}}(d z, d u)=\mathrm{e}^{\langle z, \theta(u)\rangle} \ell(d z) d u .
$$

One refers to this measure as the compensator measure of $L$. Since the compensator measure is time-dependent, but deterministic, $L$ is an independent increment process under $\widetilde{P}$. If $\theta_{1}, \theta_{2}$ are supposed to be constant, then $L$ will have stationary increments under $\widetilde{P}$, and therefore becomes a $\widetilde{P}$-Lévy process in that case. We remark in passing that we could use the Esscher transform to change measure for the subordinators driving the stochastic volatility model discussed in Section 3 , and thereby modelling the market price of volatility risk. 
Before analysing $p(t, T, K)$ further, let us discuss the function $F(a, b, K ; x)$ defined in Prop. 4.2. Recall from the proof of Prop. 4.2 that we can express $F$ as

$$
F(a, b, K ; x)=\mathbb{E}\left[(\exp (a+b U+x)-K)^{+}\right],
$$

where $U$ is a standard normally distributed random variable. We note here that $b$ is strictly positive. It is then simple to see that $\exp (-c x) F(a, b, K ; x)$ is integrable on $\mathbb{R}$ for any $c>1$, as the next Lemma proves:

Lemma 4.4. For any constant $c>1$, we have that $\exp (-c x) F(a, b, K ; x) \in L^{1}(\mathbb{R})$.

Proof. By Tonelli's theorem, it holds,

$$
\begin{aligned}
\int_{\mathbb{R}} \mathrm{e}^{-c x} F(a, b, K ; x) d x & =\mathbb{E}\left[\int_{\mathbb{R}} \mathrm{e}^{-c x}\left(\mathrm{e}^{a+b U+x}-K\right)^{+} d x\right] \\
& =\mathbb{E}\left[\int_{\ln K-a-b U}^{\infty} \mathrm{e}^{-c x}\left(\mathrm{e}^{a+b U+x}-K\right) d x\right] \\
& \leq \mathbb{E}\left[\mathrm{e}^{a+b U} \int_{\ln K-a-b U}^{\infty} \mathrm{e}^{-(c-1) x} d x\right] \\
& =\frac{1}{c-1} \mathrm{e}^{a-(c-1)(\ln K-a)} \mathbb{E}\left[\mathrm{e}^{b c U}\right]<\infty,
\end{aligned}
$$

where in the last step we have used that a standard normal distributed random variable has finite exponential moments of all orders. Hence, the Lemma follows.

In the next Lemma we find the Fourier transform of the function $\exp (-c x) F(a, b, K ; x)$ for $c>1$. For this purpose, we apply the definition of the Fourier transform of a function $g \in L^{1}(\mathbb{R})$ given in Folland [16];

$$
\widehat{g}(y)=\int_{\mathbb{R}} g(x) \mathrm{e}^{-\mathrm{i} x y} d x .
$$

Notice the sign in the complex exponent. With this definition, it holds that the inverse Fourier transform can be expressed as

$$
g(x)=\frac{1}{2 \pi} \int_{\mathbb{R}} \widehat{g}(y) \mathrm{e}^{\mathrm{i} x y} d y
$$

as long as $\widehat{g} \in L^{1}(\mathbb{R})$.

Lemma 4.5. The Fourier transform of $F_{c}(a, b, K ; x):=\exp (-c x) F(a, b, K ; x)$ is

$$
\widehat{F}_{c}(a, b, K ; y)=\frac{\mathrm{e}^{a-(\mathrm{i} y+(c-1))(\ln K-a)+\frac{1}{2} b^{2}(1+(\mathrm{i} y+(c-1)))^{2}}}{(c-1)+\mathrm{i} y}-K \frac{\mathrm{e}^{-(\mathrm{i} y+c)(\ln K-a)+\frac{1}{2} b^{2}(\mathrm{i} y+c)^{2}}}{c+\mathrm{i} y},
$$

for every $c>1$.

Proof. By the Fubini-Tonelli Theorem, we compute as follows:

$$
\begin{aligned}
\int_{\mathbb{R}} \mathrm{e}^{-c x} F(a, b . K ; x) \mathrm{e}^{-\mathrm{i} x y} d x=\mathbb{E} & {\left[\int_{\mathbb{R}} \mathrm{e}^{-c x}\left(\mathrm{e}^{a+b U+x}-K\right)^{+} \mathrm{e}^{-\mathrm{i} x y} d x\right] } \\
=\mathbb{E} & {\left[\mathrm{e}^{a+b U} \int_{\ln K-a-b U}^{\infty} \mathrm{e}^{-((c-1)+\mathrm{i} y) x} d x\right] } \\
& -K \mathbb{E}\left[\int_{\ln K-a-b U}^{\infty} \mathrm{e}^{-(c+\mathrm{i} y) x} d x\right] .
\end{aligned}
$$

Hence, the Lemma follows after a straightforward integration of exponentials.

Note that the Fourier transformed function $\widehat{F}_{c}(a, b, K ; \cdot) \in L^{1}(\mathbb{R})$ since both terms will consist of expressions involving $\exp \left(-b^{2} y^{2} / 2\right)$. Hence, using the inverse Fourier transform, we find

$$
F(a, b, K ; x)=\frac{1}{2 \pi} \int_{\mathbb{R}} \widehat{F}_{c}(a, b, K ; y) \mathrm{e}^{(\mathrm{i} y+c) x} d y .
$$

We are ready for our next proposition on the derivation of $p(t, T, K)$ in (4.9): 
Proposition 4.6. For a given $c>1$, suppose that $(c, c+1) \in R$. Then it holds that

$$
\begin{aligned}
p(t, T, K)=\exp & \left(\frac{\sigma_{2}^{2}}{4 \alpha_{2}}\left(1-\mathrm{e}^{-2 \alpha_{2}(T-t)}\right)\right) \\
& \times \frac{1}{2 \pi} \int_{\mathbb{R}} \widehat{F}_{c}(a(t, T), \Sigma(t, T), K ; y) \exp (\Phi(T-t, y)) d y,
\end{aligned}
$$

where $a(t, T)$ and $\Sigma(t, T)$ are defined in Prop. 4.2, and $\Phi(\tau, y)$ is given by

$$
\Phi(\tau, y)=\int_{0}^{\tau} \phi\left((\mathrm{i} y+c) \mathrm{e}^{-\beta_{1} u},(\mathrm{i} y+c+1) \mathrm{e}^{-\beta_{2} u}\right) d u
$$

Proof. First, define the density process

$$
Z(t)=\exp \left(\int_{0}^{t} \mathrm{e}^{-\beta_{2}(T-u)} d L_{2}(u)-\int_{0}^{t} \phi\left(0, \mathrm{e}^{-\beta_{2}(T-u)}\right) d u\right),
$$

corresponding to letting $\theta_{1}(u)=0$ and $\theta_{2}(u)=\exp \left(-\beta_{2}(T-u)\right)$ in the Esscher transform defined above. According the Lemma 4.3, this is a measure transform giving rise to an equivalent probability $P_{\beta}$ such that $\left(L_{1}, L_{2}\right)$ becomes an independent increment process under $P_{\beta}$, with explicitly known conditional cumulant function. Observe that

$$
\frac{Z(T)}{Z(t)}=\exp \left(\Psi_{2}(t, T)-\int_{t}^{T} \phi\left(0, \mathrm{e}^{-\beta_{2}(T-u)}\right) d u\right) .
$$

Denoting $\mathbb{E}_{\beta}$ the expectation operator under $P_{\beta}$, we find from the Fourier inversion formula and Fubini-Tonelli,

$$
\begin{aligned}
p(t, T, K)= & \exp \left(\frac{\sigma_{2}^{2}}{4 \alpha_{2}}\left(1-\mathrm{e}^{-2 \alpha_{2}(T-t)}\right)+\int_{0}^{T-t} \phi\left(0, \mathrm{e}^{-\beta_{2} u}\right) d u\right) \\
& \times \mathbb{E}_{\beta}\left[F\left(a(t, T), \Sigma(t, T), K ; \Psi_{1}(t, T)-\Psi_{2}(t, T)\right)\right] \\
= & \exp \left(\frac{\sigma_{2}^{2}}{4 \alpha_{2}}\left(1-\mathrm{e}^{-2 \alpha_{2}(T-t)}\right)+\int_{0}^{T-t} \phi\left(0, \mathrm{e}^{-\beta_{2} u}\right) d u\right) \\
& \times \frac{1}{2 \pi} \int_{\mathbb{R}} \widehat{F}_{c}(a(t, T), \Sigma(t, T), K ; y) \mathbb{E}_{\beta}\left[\exp \left((\mathrm{i} y+c)\left(\Psi_{1}(t, T)-\Psi_{2}(t, T)\right)\right] d y,\right.
\end{aligned}
$$

with $a(t, T)$ and $\Sigma(t, T)$ defined in Prop. 4.2. But, appealing to the definition of the measure $P_{\beta}$, we find

$$
\begin{aligned}
\ln \mathbb{E}_{\beta}\left[\exp \left((\mathrm{i} y+c)\left(\Psi_{1}(t, T)-\Psi_{2}(t, T)\right)\right)\right]=-\int_{0}^{T-t} \phi\left(0, \mathrm{e}^{-\beta_{2} u}\right) d u \\
\quad+\ln \mathbb{E}\left[\exp \left(\int_{t}^{T}(\mathrm{i} y+c) \mathrm{e}^{-\beta_{1}(T-u)} d L_{1}(u)+\int_{t}^{T}(\mathrm{i} y+c+1) \mathrm{e}^{-\beta_{2}(T-u)} d L_{2}(u)\right)\right] \\
=-\int_{0}^{T-t} \phi\left(0, \mathrm{e}^{-\beta_{2} u}\right) d u+\int_{t}^{T} \phi\left((\mathrm{i} y+c) \mathrm{e}^{-\beta_{1}(T-u)},(\mathrm{i} y+c+1) \mathrm{e}^{-\beta_{2}(T-u)}\right) d u
\end{aligned}
$$

where we used the independent increment property of the Lévy process $L$. But then the result follows.

We collect everything together, to state the Margrabe formula for energy spread options:

Theorem 4.7. Suppose for a $c>1$ that $(c, c+1) \in R$ in condition (4.5). Then the price $P(t)$ for the spread option is given by

$$
P(t)=\mathrm{e}^{-r(T-t)} C_{1}\left(t, T, X_{1}(t), Y_{1}(t)\right) p(t, T, K)
$$

where $p(t, T, K)$ is given in Prop. 4.6 and

$$
K=h \frac{C_{2}\left(t, T, X_{2}(t), Y_{2}(t)\right)}{C_{1}\left(t, T, X_{1}(t), Y_{1}(t)\right)}
$$

with $C_{i}\left(t, T, x_{i}, y_{i}\right), i=1,2$ defined in (4.6). 
Let us discuss the asymptotic properties of this generalization of Margrabe's formula. Indeed, from the definition of $C_{i}\left(t, T, x_{i}, y_{i}\right), i=1,2$ defined in (4.6), we find that

$$
C_{i}(t, T, x, y) \sim \Lambda_{i}(T) \exp \left(\frac{\mu_{i}}{\alpha_{i}}+\frac{\gamma_{i}}{\beta_{i}}\right),
$$

when $T-t$ tends to infinity. This means that the strike $K$ behaves asymptotically for maturities far in the future as

$$
K \sim h \frac{\Lambda_{2}(T)}{\Lambda_{1}(T)} \exp \left(\frac{\mu_{2}}{\alpha_{2}}-\frac{\mu_{1}}{\alpha_{1}}+\frac{\gamma_{2}}{\beta_{2}}-\frac{\gamma_{1}}{\beta_{1}}\right) .
$$

Furthermore, from Prop. 4.6 and supposing natural integrability hypotheses, we find after letting $T-t \rightarrow \infty$

$$
p(t, T, K) \sim \frac{1}{2 \pi} \mathrm{e}^{\frac{\sigma_{2}^{2}}{4 \alpha_{2}}} \int_{\mathbb{R}} \widehat{F}_{c}(\widetilde{a}, \widetilde{\Sigma}, K ; y) \exp (\widetilde{\Phi}(y)) d y,
$$

with

and

$$
\widetilde{a}=\rho \frac{\sigma_{1} \sigma_{2}}{\alpha_{1}+\alpha_{2}}-\frac{\sigma_{2}^{2}}{2 \alpha_{2}},
$$

Moreover,

$$
\widetilde{\Sigma}=\frac{\sigma_{1}^{2}}{2 \alpha_{1}}-2 \rho \frac{\sigma_{1} \sigma_{2}}{\alpha_{1}+\alpha_{2}}+\frac{\sigma_{2}^{2}}{2 \alpha_{2}} .
$$

$$
\widetilde{\Phi}(y)=\int_{0}^{\infty} \phi\left((\mathrm{i} y+c) \mathrm{e}^{-\beta_{1} u},(\mathrm{i} y+c+1) \mathrm{e}^{-\beta_{2} u}\right) d u .
$$

This integral is well-defined under logarithmic integrability hypothesis of the jump processes, see Sato [25]. In conclusion, we have that the option price behaves asymptotically as

$$
P(t) \sim k_{1} \mathrm{e}^{-r(T-t)} \Lambda_{1}(T) \int_{\mathbb{R}} \widehat{F}_{c}\left(\widetilde{a}, \widetilde{\Sigma}, k_{2} \frac{\Lambda_{2}(T)}{\Lambda_{1}(T)}\right) \exp (\widetilde{\Phi}(y)) d y,
$$

for constants $k_{1}$ and $k_{2}$ independent of $t$ and $T$. The option prices will not be influenced by the current spot price levels when we are far from exercise. This is an effect of the stationary processes driving the spot dynamics.

4.2. Computing sensitivity measures of cross-commodity options. With the (semi-)explicit price for the spread option in Theorem 4.7 at hand, one can start to derive the "Greeks" for risk management purposes. By inspecting the price, we see that to find the Greek of $P(t)$ with respect to $X_{1}(t)$, say, will involve differentiation of the function $C_{1}\left(t, T, X_{1}(t), Y_{1}(t)\right)$, which appears both explicitly and inside the inverse Fourier transform in the expression of the strike price $K$. To differentiate inside the inverse Fourier transform would yield an expression analogous to differentiating first the payoff function of the derivative and then apply Fourier methods to compute the resulting expectation.

In this Subsection we want to investigate a different approach based on the so-called density method (see Glasserman [17]). The density method allows for differentiation of option prices for many particular models, where one does not need to differentiate the payoff function. Our analysis will be valid for a rather general class of cross-commodity options, that is, European-style options written on the underlying bivariate commodity prices $\left(S_{1}, S_{2}\right)$. Noting that each of the two price processes has a Brownian motion driven factor, one can exploit this by a conditioning argument to obtain expressions for the derivatives with respect to all four factors $X_{i}(t), Y_{j}(t), i, j=1,2$. This approach, called the conditional density method, was suggested and analysed for options written on one underlying asset in Benth, Di Nunno and Khedher [6]. It was later extended to multi-factor models and applied to energy markets in Benth, Di Nunno and Khedher [7]. We apply it here in our particular multi-factor cross commodity model, but remark that its potential is much larger.

Consider a cross commodity option paying $\widetilde{g}\left(S_{1}(T), S_{2}(T)\right.$ ) at time $T$, for some (nice) function $\widetilde{g}: \mathbb{R}_{+}^{2} \mapsto \mathbb{R}_{+}$. By a simple reformulation, we can express this payoff function as

$$
\widetilde{g}\left(S_{1}(T), S_{2}(T)\right)=g\left(T, X_{1}(T)+Y_{1}(T), X_{2}(T)+Y_{2}(T)\right),
$$


for a function $g$. From now on, we surpress the dependency on $T$ in this function (it comes from the seasonality functions), and suppose that

$$
\mathbb{E}\left[\left|g\left(X_{1}(T)+Y_{1}(T), X_{2}(T)+Y_{2}(T)\right)\right|\right]<\infty .
$$

Our problem now is to find the derivative of the price functionals

$$
P(t)=\mathrm{e}^{-r(T-t)} \mathbb{E}\left[g\left(X_{1}(T)+Y_{1}(T), X_{2}(T)+Y_{2}(T)\right) \mid \mathcal{F}_{t}\right] .
$$

For simplicity, we let $r=0$ in the rest of this Subsection.

By the Markov property of the factors, we find that

$$
P(t)=P\left(t, X_{1}(t), Y_{1}(t), X_{2}(t), Y_{2}(t)\right),
$$

where

$$
\begin{aligned}
& P\left(t, x_{1}, y_{1}, x_{2}, y_{2}\right)=\mathbb{E}\left[g \left(x_{1} \mathrm{e}^{-\alpha_{1}(T-t)}+y_{1} \mathrm{e}^{-\beta_{1}(T-t)}+\Xi_{1}(t, T)+\Psi_{1}(t, T),\right.\right. \\
&\left.\left.x_{2} \mathrm{e}^{-\alpha_{2}(T-t)}+y_{2} \mathrm{e}^{-\beta_{2}(T-t)}+\Xi_{2}(t, T)+\Psi_{2}(t, T)\right)\right],
\end{aligned}
$$

after using Lemma 4.1 and (4.7)-(4.8). By conditioning, we have the following representation:

Proposition 4.8. It holds that

$$
P\left(t, x_{1}, y_{1}, x_{2}, y_{2}\right)=\mathbb{E}\left[\int_{\mathbb{R}^{2}} g\left(z_{1}, z_{2}\right) p_{\Xi}\left(z_{1}\left(x_{1}, y_{1}\right), z_{2}\left(x_{2}, y_{2}\right)\right) d z_{1} d z_{2}\right]
$$

where

$$
z_{i}\left(x_{i}, y_{i}\right)=z_{i}-x_{i} \mathrm{e}^{-\alpha_{i}(T-t)}-y_{i} \mathrm{e}^{-\beta_{i}(T-t)}-\Psi_{i}(t, T),
$$

for $i=1,2$, and $p_{\Xi}\left(z_{1}, z_{2}\right)$ is the density function of the bivariate normal random variable $\left(\Xi_{1}(t, T), \Xi_{2}(t, T)\right)$.

Proof. First observe that by assumption, $\Psi_{i}(t, T)$ are independent of $\Xi_{i}(t, T), i=1,2$. By conditioning, we find from properties of the conditional expectation that

$$
\begin{aligned}
P\left(t, x_{1}, y_{1}, x_{2}, y_{2}\right)=\mathbb{E}\left[\mathbb { E } \left[g \left(x_{1} \mathrm{e}^{-\alpha_{1}(T-t)}+y_{1} \mathrm{e}^{-\beta_{1}(T-t)}+\psi_{1}+\Xi_{1}(t, T),\right.\right.\right. & \\
& \left.\left.\left.x_{2} \mathrm{e}^{-\alpha_{2}(T-t)}+y_{2} \mathrm{e}^{-\beta_{2}(T-t)}+\psi_{2}+\Xi_{2}(t, T)\right) \mid \psi_{i}=\Psi_{i}(t, T), i=1,2\right]\right] .
\end{aligned}
$$

We see that the inner expression is an expectation of a function of $\left(\Xi_{1}(t, T), \Xi_{2}(t, T)\right)$, and the result follows.

From the definition of $\Xi_{i}(t, T), i=1,2$, in (4.7) we find that it has expected value zero and variance given by the Ito isometry as

$$
v_{i}(T-t) \triangleq \mathbb{E}\left[\left(\int_{t}^{T} \sigma_{i} \mathrm{e}^{-\alpha_{i}(T-s)} d B_{i}(s)\right)^{2}\right]=\frac{\sigma_{i}^{2}}{2 \alpha_{i}}\left(1-\mathrm{e}^{-2 \alpha_{i}(T-t)}\right) .
$$

Furthermore, since $B_{1}$ and $B_{2}$ are two correlated Brownian motion, the Ito isometry yields the covariance between $\Xi_{1}(t, T)$ and $\Xi_{2}(t, T)$ as

$$
v_{12}(T-t) \triangleq \mathbb{E}\left[\Xi_{1}(t, T) \Xi_{2}(t, T)\right]=\rho \frac{\sigma_{1} \sigma_{2}}{\alpha_{1}+\alpha_{2}}\left(1-\mathrm{e}^{-\left(\alpha_{1}+\alpha_{2}\right)(T-t)}\right) .
$$

This gives a full specification of the variance-covariance matrix $V(T-t) \in \mathbb{R}^{2 \times 2}$ of $\left(\Xi_{1}(t, T), \Xi_{2}(t, T)\right)$, and its bivariate probability density becomes

$$
p_{\Xi}\left(z_{1}, z_{2}\right)=\frac{1}{2 \pi \sqrt{\operatorname{det}(V(T-t))}} \exp \left(-\frac{1}{2} z^{*} V^{-1}(T-t) z\right),
$$

with $z=\left(z_{1}, z_{2}\right)^{*}$ and $*$ meaning the transpose. It is easily seen that the gradient of $p_{\Xi}$ is

$$
\nabla p_{\Xi}\left(z_{1}, z_{2}\right)=-p_{\Xi}\left(z_{1}, z_{2}\right)\left(\mathbf{e}_{1}^{*} V^{-1} z, \mathbf{e}_{2}^{*} V^{-1} z\right)
$$

where $\mathbf{e}_{i}, i=1,2$, are the Euclidean basis vectors in $\mathbb{R}^{2}$. We are now ready to derive the sensitivity of the option price with respect to the various factors. 
For the sake of illustration, suppose we want to find the derivative of the option price with respect to $X_{1}(t)$, the first factor of the first commodity (energy) in the option. This is given via the derivative $\partial P\left(t, x_{1}, y_{1}, x_{2}, y_{2}\right) / \partial x_{1}$. By Prop. 4.8 this is now straightforwardly calculated. We find

$$
\begin{aligned}
\frac{P\left(t, x_{1}, y_{1}, x_{2}, y_{2}\right)}{\partial x_{1}}=\mathbb{E}\left[\int_{\mathbb{R}^{2}} g\left(z_{1}, z_{2}\right) p_{\Xi}\left(z_{1}\left(x_{1}, y_{1}\right), z_{2}\left(x_{2}, y_{2}\right)\right)\right. \\
\left.\quad \times \mathbf{e}_{1}^{*} V^{-1}\left(z_{1}\left(x_{1}, y_{1}\right), z_{2}\left(x_{2}, y_{2}\right)\right)^{*} \mathrm{e}^{-\alpha_{1}(T-t)} d z_{1} d z_{2}\right]
\end{aligned}
$$

According to Folland [16], Thm. 2.27, we can commute differentiation and integration in the above derivation as long as the integrand in (4.21) has a majorization uniformly in $x_{1}$. But this holds at least when restricting $x_{1}$ to a bounded subset of $\mathbb{R}$. Tracing back, we get

$$
\begin{aligned}
& \frac{P\left(t, x_{1}, y_{1}, x_{2}, y_{2}\right)}{\partial x_{1}} \\
& =\mathrm{e}^{-\alpha_{1}(T-t)} \mathbb{E}\left[g \left(x_{1} \mathrm{e}^{-\alpha_{1}(T-t)}+y_{1} \mathrm{e}^{-\beta_{1}(T-t)}+\Xi_{1}(t, T)+\Psi_{1}(t, T),\right.\right. \\
& \left.\left.\quad x_{2} \mathrm{e}^{-\alpha_{2}(T-t)}+y_{2} \mathrm{e}^{-\beta_{2}(T-t)}+\Xi_{2}(t, T)+\Psi_{2}(t, T)\right) \mathbf{e}_{1}^{*} V^{-1}\left(\Xi_{1}(t, T), \Xi_{2}(t, T)\right)^{*}\right] .
\end{aligned}
$$

Observe that the above Greek does not involve any differentiation of the payoff function $g$, and lends itself easily to Monte Carlo pricing. In fact, we are almost back to pricing the option itself, except for the additional weight functional

$$
\mathbf{e}_{1}^{*} V^{-1}\left(\Xi_{1}(t, T), \Xi_{2}(t, T)\right)^{*},
$$

that enters the expectation operator, and a "discounting" term given by the speed of mean reversion $\alpha_{1}$.

Of course, the derivatives with respect to the other factors are calculable in the exact same fashion. Indeed, we can also compute derivatives with respect to some of the parameters, like for example the speeds of mean reversion using this approach. This would, however, yield more technically complex expressions, and we refrain from analyzing this further here.

4.3. Cross-commodity dependency risk and copulas. In this Subsection we consider the dynamics (4.3)-(4.4) defined under the market probability $P$, and focus on how to do a measure change from $P$ to $Q$ for bivariate jump process. In particular, we are interested in the potential effects on the dependency structure.

The Esscher transform (as previously introduced) is the standard approach to produce a parametric class of measure changes for jump processes. For the Brownian motions, one naturally applies the Girsanov theorem to change measure. To this end, we suppose that $\theta=\left(\theta_{1}, \theta_{2}\right) \in \mathbb{R}^{2}$ and $\eta=\left(\eta_{1}, \eta_{2}\right) \in \mathbb{R}^{2}$ are two constant vectors. Define the martingale process $Z_{B}(t)$ for $t \leq T$

$$
Z_{B}(t)=\exp \left(\theta_{1} B_{1}(t)+\theta_{2} B_{2}(t)-\frac{1}{2}\left(\theta_{1}^{2}+\theta_{2}^{2}\right) t\right) .
$$

Then, by Girsanov's Theorem, we find that $Z(t)$ is the density process of an equivalent probability measure $Q_{B}$ such that

$$
d W_{i}(t)=d B_{i}(t)-\theta_{i} d t, i=1,2,
$$

are $Q_{B}$-Brownian motions on $[0, T]$. Observe that the correlation between $B_{1}$ and $B_{2}$ is preserved under this measure change, so that $W_{1}$ and $W_{2}$ also become correlated by the same factor $\rho$. The characteristics of the Lévy processes $L_{1}, L_{2}$ remain unchanged under $Q_{B}$.

Recall the Esscher transform defining a measure via a density process $Z(t)$ as in (4.10). We shall here focus on a constant measure change, and define the process $Z_{L}(t)$ for $t \leq T$ as

$$
Z_{L}(t)=\exp \left(\eta_{1} L_{1}(t)+\eta_{2} L_{2}(t)-\phi\left(\eta_{1}, \eta_{2}\right) t\right)
$$

where $\phi(x, y)$ is the logarithm of the moment generating function of the bivariate random variable $\left(L_{1}(1), L_{2}(1)\right)$. Choosing $\eta=\left(\eta_{1}, \eta_{2}\right) \in R$ (see (4.5)) implies that $Z_{L}(t)$ is a martingale with expectation equal to one, and therefore the density process of an equivalent probability $Q_{L}$. This measure transform of $\left(L_{1}(t), L_{2}(t)\right)$ preserves the Lévy property of $\left(L_{1}(t), L_{2}(t)\right)$ (recall Lemma 4.3 
above). The change of measure from $P$ to $Q_{L}$ does not affect the Brownian part since the jump process and the Brownian motions are supposed independent.

We characterize the Lévy process $\left(L_{1}(t), L_{2}(t)\right)$ under $Q_{L}$. By a direct computation, we find the logarithm of the moment generating function to be

$$
\begin{aligned}
\phi_{Q}(x, y) & =\ln \mathbb{E}_{Q_{L}}\left[\exp \left(x L_{1}(1)+y L_{2}(1)\right)\right] \\
& =\ln \mathbb{E}\left[\exp \left(\left(x+\eta_{1}\right) L_{1}(1)+\left(y+\eta_{2}\right) L_{2}(1)\right)\right]-\phi\left(\eta_{1}, \eta_{2}\right) \\
& =\phi\left(x+\eta_{1}, y+\eta_{2}\right)-\phi\left(\eta_{1}, \eta_{2}\right) .
\end{aligned}
$$

To make this derivation rigorous, we must assume that $\left(x+\eta_{1}, y+\eta_{2}\right) \in R$. If $L(t)$ has drift given by the vector $\xi \in \mathbb{R}^{2}$ and a Lévy measure denoted by $\ell\left(d z_{1}, d z_{2}\right)$, we find from the Lévy-Kintchine representation that

$$
\begin{aligned}
\phi_{Q}(x, y)=\xi_{1} x & +\xi_{2} y+\int_{|z|<1}\left(x z_{1}+y z_{2}\right)\left(\mathrm{e}^{\eta_{1} z_{1}+\eta_{2} z_{2}}-1\right) \ell\left(d z_{1}, d z_{2}\right) \\
& +\int_{\mathbb{R}_{0}} \int_{\mathbb{R}_{0}}\left\{\mathrm{e}^{x z_{1}+y z_{2}}-1-\mathbf{1}_{\{|z|<1\}}\left(x z_{1}+y z_{2}\right)\right\} \ell\left(d z_{1}, d z_{2}\right)
\end{aligned}
$$

where $|\cdot|$ is the norm on $\mathbb{R}^{2}$. Hence, the drift of $L=\left(L_{1}, L_{2}\right)$ is

$$
\left(\xi_{1}+\int_{|z|<1} z_{1}\left\{\mathrm{e}^{\eta_{1} z_{1}+\eta_{2} z_{2}}-1\right\} \ell\left(d z_{1}, d z_{2}\right), \xi_{2}+\int_{|z|<1} z_{2}\left\{\mathrm{e}^{\eta_{1} z_{1}+\eta_{2} z_{2}}-1\right\} \ell\left(d z_{1}, d z_{2}\right)\right)
$$

under $Q_{L}$, whereas the Lévy measure is

$$
\ell_{Q}\left(d z_{1}, d z_{2}\right)=\mathrm{e}^{\eta_{1} z_{1}+\eta_{2} z_{2}} \ell\left(d z_{1}, d z_{2}\right) .
$$

We see that the effect of the Esscher transform is a linear shift in the drift and an exponential tilting of the Lévy measure.

We define a pricing measure $Q \sim P$ as $Q=Q_{B} \times Q_{L}$, which then will have a Radon-Nikodym derivative with density

$$
\left.\frac{d Q}{d P}\right|_{\mathcal{F}_{t}}=Z_{B}(t) Z_{L}(t)
$$

for $t \leq T$. We know the characteristics of $L$ and $B_{1}, B_{2}$ under $Q$.

We next compute the forward price dynamics for a contract delivering the spot at time $T$. By definition, we set the forward price at time $t \leq T$ on commodity $i$, denoted $f_{i}(t, T)$, as

$$
f_{i}(t, T)=\mathbb{E}_{Q}\left[S_{i}(T) \mid \mathcal{F}_{t}\right] .
$$

Proposition 4.9. Suppose that $\left(\eta_{1}+1, \eta_{2}+1\right) \in R$. Then, the forward prices $f_{i}(t, T), i=1,2$, are given by

$$
f_{i}(t, T)=\Lambda_{i}(T) \exp \left(X_{i}(t) \mathrm{e}^{-\alpha_{i}(T-t)}+Y_{i}(t) \mathrm{e}^{-\beta_{i}(T-t)}\right) \Upsilon_{i}(T-t) \Theta_{i}(T-t),
$$

where

$$
\ln \Upsilon_{i}(u)=\frac{\mu_{i}}{\alpha_{i}}\left(1-\mathrm{e}^{-\alpha_{i} u}\right)+\frac{\gamma_{i}}{\beta_{i}}\left(1-\mathrm{e}^{-\beta_{i} u}\right)+\frac{\sigma_{i}^{2}}{4 \alpha_{i}}\left(1-\mathrm{e}^{-2 \alpha_{i} u}\right)
$$

and

$$
\ln \Theta_{i}(u)=\frac{\sigma_{i} \theta_{i}}{\alpha_{i}}\left(1-\mathrm{e}^{-\alpha_{i} u}\right)+\int_{0}^{u} \phi\left(\eta_{1}+\mathbf{1}_{\{i=1\}} \mathrm{e}^{-\beta_{1} v}, \eta_{2}+\mathbf{1}_{\{i=2\}} \mathrm{e}^{-\beta_{2} v}\right)-\phi\left(\eta_{1}, \eta_{2}\right) d v,
$$

with $0 \leq u \leq T$.

Proof. From Lemma 4.1 and adaptedness of $X_{i}(t)$ and $Y_{i}(t)$ to $\mathcal{F}_{t}$ we find

$$
\begin{aligned}
f_{i}(t, T)=\Lambda_{i} & (T) \exp \left(X_{i}(t) \mathrm{e}^{-\alpha_{i}(T-t)}+Y_{i}(t) \mathrm{e}^{-\beta_{i}(T-t)}\right) \\
& \times \exp \left(\frac{\mu_{i}+\sigma_{i} \theta_{i}}{\alpha_{i}}\left(1-\mathrm{e}^{-\alpha_{i} u}\right)+\frac{\gamma_{i}}{\beta_{i}}\left(1-\mathrm{e}^{-\beta_{i} u}\right)\right)
\end{aligned}
$$




$$
\times \mathbb{E}_{Q}\left[\exp \left(\int_{t}^{T} \sigma_{i} \mathrm{e}^{-\alpha_{i}(T-v)} d W_{i}(v)+\int_{t}^{T} \mathrm{e}^{-\beta_{i}(T-v)} d L_{i}(v)\right) \mid \mathcal{F}_{t}\right] .
$$

Focusing on the conditional expectation, we first recall that $W_{i}$ and $L_{i}$ have independent increments under $Q$, and moreover are independent. Hence,

$$
\begin{aligned}
\mathbb{E}_{Q} & {\left[\exp \left(\int_{t}^{T} \sigma_{i} \mathrm{e}^{-\alpha_{i}(T-v)} d W_{i}(v)+\int_{t}^{T} \mathrm{e}^{-\beta_{i}(T-v)} d L_{i}(v)\right) \mid \mathcal{F}_{t}\right] } \\
& =\mathbb{E}_{Q_{B}}\left[\exp \left(\int_{t}^{T} \sigma_{i} \mathrm{e}^{-\alpha_{i}(T-v)} d W_{i}(v)\right)\right] \times \mathbb{E}_{Q_{L}}\left[\exp \left(\int_{t}^{T} \mathrm{e}^{-\beta_{i}(T-v)} d L_{i}(v)\right)\right] .
\end{aligned}
$$

The first expectation is simple to compute after observing that the Wiener integral is normally distributed. Hence,

$$
\mathbb{E}_{Q_{B}}\left[\exp \left(\int_{t}^{T} \sigma_{i} \mathrm{e}^{-\alpha_{i}(T-v)} d W_{i}(v)\right)\right]=\exp \left(\frac{\sigma_{i}^{2}}{4 \alpha_{i}}\left(1-\mathrm{e}^{-2 \alpha_{i}(T-t)}\right)\right) .
$$

The last expectation is computed by appealing to the measure change $Q_{L}$ and the definition of the logarithm of the cumulant function

$$
\begin{aligned}
\mathbb{E}_{Q_{L}}\left[\exp \left(\int_{t}^{T} \mathrm{e}^{-\beta_{i}(T-v)} d L_{i}(v)\right)\right] \\
=\mathbb{E}\left[\exp \left(\int_{t}^{T} \mathrm{e}^{-\beta_{i}(T-v)} d L_{i}(v)+\eta_{1}\left(L_{1}(T)-L_{1}(t)\right)+\eta_{2}\left(L_{2}(T)-L_{2}(t)\right)\right)\right] \\
\times \exp \left(-\phi\left(\eta_{1}, \eta_{2}\right)(T-t)\right) .
\end{aligned}
$$

Observe that we must require that $\left(\eta_{1}+\exp \left(-\beta_{1} u\right), \eta_{2}+\exp \left(-\beta_{2} u\right)\right) \in R$ for $0 \leq u \leq T$ for this to hold. However, the condition in the proposition is sufficient for this. Hence, the proof is complete.

We observe that $\theta$ and $\eta$ describe the risk premium in the sense of determining the market price of risk. For the sake of illustration, suppose that $\eta=(0,0)$, and we find that the risk premium becomes

$$
\begin{aligned}
R_{i}(t, T)=\Lambda_{i}(T) & \exp \left(X_{i}(t) \mathrm{e}^{-\alpha_{i}(T-t)}+Y_{i}(t) \mathrm{e}^{-\beta_{i}(T-t)}\right) \Upsilon_{i}(T-t) \\
& \times\left\{\exp \left(\frac{\sigma_{i} \theta_{i}}{\alpha_{i}}\left(1-\mathrm{e}^{-\alpha_{i}(T-t)}\right)\right)-1\right\}
\end{aligned}
$$

Hence, as $\sigma_{i}$, the volatility, is naturally positive, we find a positive risk premium whenever the market price of risk $\theta_{i}$ is positive, and vice versa. Opposite, by setting $\theta=(0,0)$, we can obtain similar conclusions on the risk premium as a function of $\eta$. We refer to Benth and Sgarra [12] for a detailed analysis of the jump market price of risk. In fact, they show that in the case of seasonally occuring spikes, one may obtain a change in the sign of the risk premium from positive to negative. We refer to [12] for details.

We specialize our discussion next to compound Poisson processes, and investigate their dependency structure in light of our measure transform. As we shall see, there is an effect on the dependency structure when using the Esscher transform, contrary to what we find for the Girsanov transform.

Let $N(t)$ be a Poisson processes with intensity $\lambda>0$, and $\left(J^{1}, J^{2}\right)$ a bivariate random variable with $\left(J_{i}^{1}, J_{i}^{2}\right), i=1, \ldots$ being independent copies of it. Let $F_{i}(x)$ be the probability distribution function of $J^{i}, i=1,2$. Define the compound Poisson processes

$$
L_{1}(t)=\sum_{i=1}^{N(t)} J_{i}^{1}
$$




$$
L_{2}(t)=\sum_{i=1}^{N(t)} J_{i}^{2} .
$$

Suppose that the distribution function of $\left(J^{1}, J^{2}\right)$ is defined via a copula function $C$, that is,

$$
F_{1,2}(x, y)=C\left(F_{1}(x), F_{2}(y)\right) .
$$

Simply put, a copula $C$ is a bivariate uniform distribution function (see Nelsen [24] for an introduction to copulas). We assume furthermore that there exist densities $p_{1}, p_{2}$ and $c(x, y)$. As long as exponential moments exist for the jumps $\left(J^{1}, J^{2}\right)$, we find

$$
\mathbb{E}\left[\mathrm{e}^{x J^{1}+y J^{2}}\right]=\int_{\mathbb{R}_{0}^{2}} \mathrm{e}^{x z_{1}+y z_{2}} c\left(F_{1}\left(z_{1}\right), F_{2}\left(z_{2}\right)\right) p_{1}\left(z_{1}\right) p_{2}\left(z_{2}\right) d z_{1} d z_{2} .
$$

Hence, the $\log$-MGF of $\left(J^{1}, J^{2}\right)$ is

$$
\phi_{1,2}(x, y) \triangleq \ln \int_{\mathbb{R}^{2}} \mathrm{e}^{x z_{1}+y z_{2}} c\left(F_{1}\left(z_{1}\right), F_{2}\left(z_{2}\right)\right) p_{1}\left(z_{1}\right) p_{2}\left(z_{2}\right) d z_{1} d z_{2} .
$$

Since

$$
\phi(x, y)=\ln \mathbb{E}\left[\mathrm{e}^{x L_{1}(1)+y L_{2}(1)}\right]=\lambda\left(\mathrm{e}^{\phi_{1,2}(x, y)}-1\right),
$$

we find that the Lévy measure of the bivariate compound Poisson process $\left(L_{1}(t), L_{2}(t)\right)$ is

$$
\ell\left(d z_{1}, d z_{2}\right)=\lambda c\left(F_{1}\left(z_{1}\right), F_{2}\left(z_{2}\right)\right) p_{1}\left(z_{1}\right) p_{2}\left(z_{2}\right) d z_{1} d z_{2} .
$$

On the other hand, one finds that the log-MGF of $\left(L_{1}(t), L_{2}(t)\right)$ under $Q$ is

$$
\phi_{Q}(x, y)=\lambda \int_{\mathbb{R}^{2}}\left(\mathrm{e}^{x z_{1}+y z_{2}}-1\right) \mathrm{e}^{\eta_{1} z_{1}+\eta_{2} z_{2}} F_{1,2}\left(d z_{1}, d z_{2}\right) .
$$

Now, introduce the probability densities

$$
\begin{aligned}
& p_{1}^{Q}(x) \triangleq k_{1}^{-1} \mathrm{e}^{\eta_{1} x} p_{1}(x) \\
& p_{2}^{Q}(y) \triangleq k_{2}^{-1} \mathrm{e}^{\eta_{2} y} p_{2}(y),
\end{aligned}
$$

with $k_{1}$ and $k_{2}$ being normalizing constants. Letting

$$
\lambda_{Q} \triangleq \lambda k_{1} k_{2}
$$

we find the Lévy measure of $L$ under $Q$ to be

$$
\ell_{Q}\left(d z_{1}, d z_{2}\right)=\lambda_{Q} c\left(F_{1}\left(z_{1}\right), F_{2}\left(z_{2}\right)\right) p_{1}^{Q}\left(z_{1}\right) p_{2}^{Q}\left(z_{2}\right) d z_{1} d z_{2} .
$$

Finally, introduce the copula density under $Q$ as

$$
c_{Q}(x, y) \triangleq c\left(F_{1}\left(\left\{F_{1}^{Q}\right\}^{-1}(x)\right), F_{2}\left(\left\{F_{2}^{Q}\right\}^{-1}(y)\right)\right)
$$

Then,

$$
\ell_{Q}\left(d z_{1}, d z_{2}\right)=\lambda_{Q} c_{Q}\left(F_{1}^{Q}\left(z_{1}\right), F_{2}^{Q}\left(z_{2}\right)\right) p_{1}^{Q}\left(z_{1}\right) p_{2}^{Q}\left(z_{2}\right) d z_{1} d z_{2} .
$$

From this we conclude that $\left(L_{1}(t), L_{2}(t)\right)$ is a bivariate compound Poisson process under $Q$ with jump intensity $\lambda_{Q}$, and $\left(J^{1}, J^{2}\right)$ having a distribution given by

$$
F_{1,2}^{Q}(x, y)=C_{Q}\left(F_{1}^{Q}(x), F_{2}^{Q}(y)\right),
$$

where $C_{Q}(x, y)$ is the copula with density $c_{Q}(x, y)$.

Let us investigate a simple example: assume that $J^{1}$ and $J^{2}$ are marginally exponentially distributed with means $1 / a$ and $1 / b$, resp., $a, b>0$. Then, from the analysis above we find

$$
\begin{aligned}
& F_{1}^{Q}(x)=1-\mathrm{e}^{-\left(a-\eta_{1}\right) x} \\
& F_{2}^{Q}(y)=1-\mathrm{e}^{-\left(b-\eta_{2}\right) y},
\end{aligned}
$$

and

$$
c_{Q}(x, y)=c\left(1-\exp \left(\frac{a}{a-\eta_{1}} \ln (1-x)\right), 1-\exp \left(\frac{b}{b-\eta_{2}} \ln (1-y)\right)\right)
$$




$$
=c\left(1-(1-x)^{a /\left(a-\eta_{1}\right)}, 1-(1-y)^{b /\left(b-\eta_{2}\right)}\right) .
$$

Hence, the density $c^{Q}$ is nothing but a nonlinear transformation of the coordinates of $c$. The effect the change of measure on the copula density is to move the mass of $c$ from coordinate $(x, y)$ to the coordinate $\left(1-(1-x)^{a / a-\eta_{1}}, 1-(1-y)^{b / b-\eta_{2}}\right)$. The function $g(x)=1-(1-x)^{q}$ for $q>0$ is monotonely increasing with $g(0)=0$ and $g(1)=1$. It holds that $g^{\prime}(0)=q$, while $g^{\prime}(1)=0$ as long as $q>1$ and $g^{\prime}(1)=+\infty$ for $q<1$. Obviously, the case $q=1$ yields the identity mapping $g(x)=x$. Since $g(x)$ is concave for $q>1$, it holds that $q(x) \geq x$ for $x \in[0,1]$. Hence, if $a / a-\eta_{1}>1$, then the first coordinate of the copula density $c$ is pushed towards higher values after transforming to the pricing measure $Q$. For $0<q<1$ we find $g(x) \leq x$, and the first coordinate of the copula density $c$ is pushed to lower values when $0<a / a-\eta_{1}<1$. For example, if $a / a-\eta_{1}>1$ and $b / b-\eta_{2}>1$, we move the mass of $c$ at a point $(x, y)$ towards the point $(1,1)$, meaning that we obtain more big jumps appearing together. By making $\eta_{1}$ and $\eta_{2}$ close to $a$ and $b$, resp., we can obtain a concentration of extreme tail dependency in the copula density $c_{Q}$. Hence, under $Q$ we get both an emphasize on bigger marginal jumps, but they will also appear more often together. Interpreted in a power market context, this means that positive market risk premia $\eta_{1}$ and $\eta_{2}$ will lead to more spikes, occuring more often in both markets at the same time, compared to the situation under the physical probability $P$. If, on the other hand, we choose $\eta_{1}$ and $\eta_{2}$ negative, we can reduce any tail dependency of $c$ under $Q$, since in this case $a / a-\eta_{1}$ and $b / b-\eta_{2}$ will become less than 1 , and the mass of $c$ at $(x, y)$ will be moved towards the origin $(0,0)$. In a power market, this would mean that under the pricing measure, we get less occurence of spikes, being reduced in size marginally, and at the same time the spikes in the two markets will become more decoupled.

In order to gain further understanding, we include a numerical example using the Gumbel copula. The Gumbel copula is defined as a parametric class of copula functions given as

$$
C_{\gamma}(x, y)=\exp \left(-\left((-\ln x)^{\gamma}+(-\ln y)^{\gamma}\right)^{1 / \gamma}\right)
$$

for $\gamma \geq 1$. The density is directly computable as

$$
\begin{aligned}
c_{\gamma}(x, y)= & C_{\gamma}(x, y) \frac{((-\ln x)(-\ln y))^{\gamma-1}}{x y}\left((-\ln x)^{\gamma}+(-\ln y)^{\gamma}\right)^{\frac{1}{\gamma}-2} \\
& \times\left\{\left((-\ln x)^{\gamma}+(-\ln y)^{\gamma}\right)^{\frac{1}{\gamma}}+(\gamma-1)\right\}
\end{aligned}
$$

In Fig. 6 we have plotted the Gumbel copula density for $\gamma=1.5$. Next, we choose jump terms in $S_{1}$ and $S_{2}$ with $a=b=0.9091$, which corresponds to an expected jump size of 1.1. Since $\exp (1.1) \approx 3.0$, we are looking at jumps which are on average scaling the price dynamics by $300 \%$, meaning spikes on average of the size of about 300\% price increase. For the sake of illustration, we use market prices of risk $\eta_{1}=\eta_{2}=0.3$. As is clear from Fig. 7, the mass of $c_{Q}$ has been transported towards $(1,1)$, yielding more emphasis on common big jumps under $Q$ than under $P$.

In the market place, there exist many swap contracts traded OTC, for example spark and dark spread swaps exchanging power with the energy equivalent of gas and coal, respectively. Furthermore, at NYMEX, one can trade in plain vanilla call and put options written on refined oil products. An analysis on the effect of $Q$ on the dependency structure is valuable for pricing purposes.

Another interesting application of the above results is the valuation of a so-called contractsfor-difference $(\mathrm{CfD})$ traded in the NordPool market. The CfD's are futures contracts written on the spread between two area prices in the Nordic market. Different spot prices for different, pre-defined, areas are the result of transmission congestions in the market, where geographical separation of production and demand is resolved by differentiation in pricing. The CfD contract is a swap, where one area price is exchanged for another, yielding a payoff at time $T$ given by $S_{1}(T)-S_{2}(T)$ for area spot prices $S_{1}$ and $S_{2}$. The CfD swap price $f_{\mathrm{CfD}}(t, T)$ at time $t \leq T$ is therefore given by

$$
f_{\mathrm{CfD}}(t, T)=\mathbb{E}_{Q}\left[S_{1}(T)-S_{2}(T) \mid \mathcal{F}_{t}\right]
$$




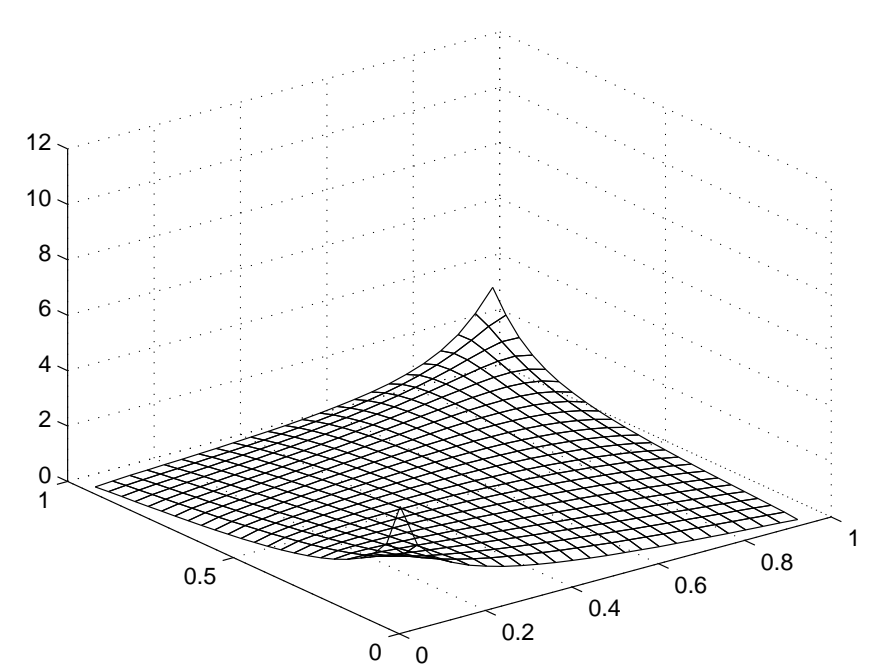

Figure 6. The Gumbel copula density function for $\gamma=1.5$.

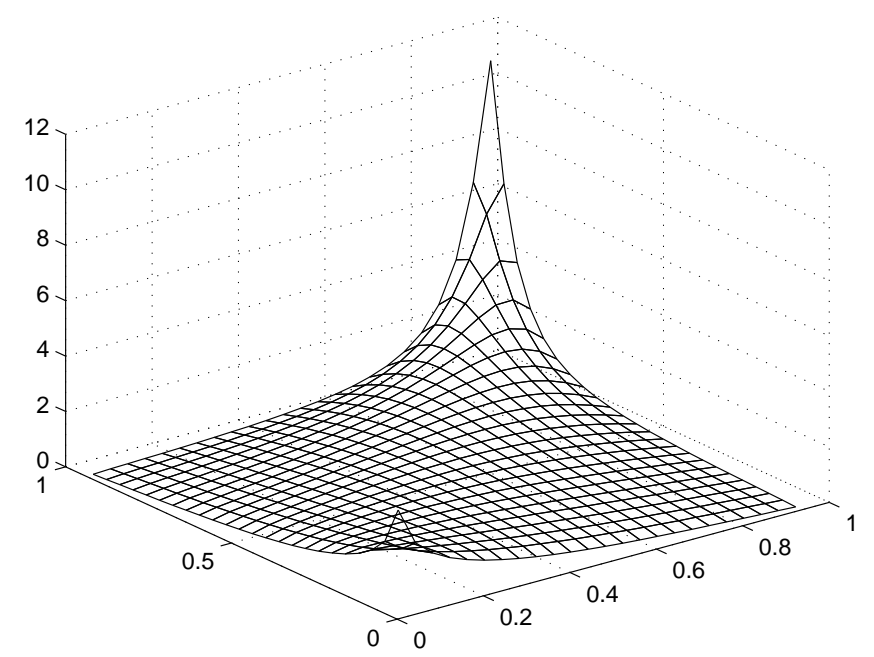

Figure 7. The Gumbel copula density function for $\gamma=1.5$ under the Esscher transform probability $Q$.

But from Prop. 4.9, we can easily derive an expression for this swap price dynamics. Recalling the discussion above with the Gumbel copula and exponentially distributed jumps, the effect of choosing an Esscher transform as the pricing measure $Q$ will be more concentration of spot prices, thus reducing the swap price volatility compared with the choice $Q=P$. It is an interesting question to look at market data for CfD swap prices together with area spot prices to see how they interact.

\section{Conclusions}

In energy markets like gas and electricity, spot prices are typically mean reverting towards a seasonal mean. Further, spikes in prices occur as a result of sudden imbalances between supply 
and demand. Stochastic volatility effects like clustering are observed as well in price changes. These stylized facts on energy spot prices call for sophisticated stochastic models.

We have proposed an exponential two-factor model with stochastic volatiliy to model energy spot prices. The deseasonalized logarithmic spot prices are governed by an Ornstein-Uhlenbeck process reverting towards a stochastic mean level, again being an Ornstein-Uhlenbeck process. The mean level is slowly varying, whereas the short term factor can typically revert faster, and thus can potentially give bigger fluctuations in prices over short time. Both Ornstein-Uhlenbeck processes are governed by correlated Brownian motions, where the short term process is assumed to have stochastic volatility defined by the Barndorff-Nielsen and Shephard model. This will enable us to model leptokurtic residuals, which is a main characteristic in energy prices.

The proposed spot price model allow for semi-analytic forward prices. In the particular case of independent Brownian motions driving the mean level and short term dynamics, the forward prices are analytic. However, the general case requires the computation of an expectation functional in the derivation of the forward price dynamics. The main complication is the dependency on an integral of the linear combination of the volatility process and its square (the variance). However, we are able to analyse properties of the forward prices, showing among other things that the forward curves allow for humps which size depends on the state of the mean-level and/or the stochastic volatility. Humps in the forward market has been observed in energy markets, for example in oil, and we provide an explanation for this by stochastic volatility and randomness in the mean level of prices.

There are close dependencies between different energy prices. For example, the electricity markets are connected to gas and coal, as these are used as fuels in power production. Different electricity markets are connected via transmission lines. We study a simplified version of our proposed spot price model set in a bivariate market context. More specifically, the marginal deseasonal logarithmic spot price dynamics is defined by a two-factor Ornstein-Uhlenbeck process driven by a Brownian motion and Lévy process. When considering two energy spot prices, we assume that both the Brownian motions and the Lévy processes are dependent. Using the Girsanov and Esscher transforms we are able to compute the price of spread options on the two energy spot prices. The price is derived based on the Fourier transform, and can be expressed in terms of the characteristics of the bivariate Lévy process. The trick in the derivation is a measure change, which reduces the bivariate option pricing problem into a problem of pricing a call option on a single underlying. Our pricing formula will extend the classical Margrabe formula for the price of a spread option on two correlated geometric Brownian motions. Sensitivity measures for the spread option is derived using a conditioning technique which exploits the independence between the Lévy processes and the Brownian motions. Spread options are traded on exchanges and bilaterally to a large extent, and their pricing and hedging is important in risk management.

The selection of the right pricing measure $Q$ is a fundamental problem in energy markets. The challenge is to find a probability $Q$ which are able to explain the risk premium. We focus on a specific issue related to this in the cross-commodity market setting, namely the question of the dependency risk premium. Looking at a bivariate compound Poisson process, we model the joint jump size distribution by a copula and analyse the change in characteristics of this particular Lévy process after performing an Esscher transform. It is known that marginally the Esscher transform will increase the spike intensity and size in the case of a positive market price of risk. We show that additionally, the jumps will happen more often in the two markets, that is, there will be a concentration of big jumps happening simultaneously in the two markets. In a numerical exercise, we illustrate this using the Gumbel copula, for which we observe an increase in the extremes for positive market prices of risk after doing an Esscher transform. These findings are of importance when pricing options on spreads and other cross-commodity derivatives.

The complexity of energy markets make them challenging to model. Cross-commodity models must account for both marginal price behaviour as well as dependencies between prices. The risk premium, in particular the dependency risk, is a delicate issue. Extensive data studies are called for to reveal the true nature of this risk premium. However, as models for the underlying spot are complex and the available data for spread options currently are rather limited, this remains a difficult task to solve. 


\section{REFERENCES}

[1] Barndorff-Nielsen, O. E., Benth, F. E., and Veraart, A. (2010). Modelling energy spot prices by Lévy semistationary processes. To appear in Bernoulli.

[2] Barndorff-Nielsen, O., and Shephard, N. (2001). Non-Gaussian Ornstein-Uhlenbeck-based models and some of their uses in economics. J. R. Statist. Soc. B, 63(2), pp. 167-241 (with discussion).

[3] Benth, F. E. (2011). The stochastic volatility model of Barndorff-Nielsen and Shephard in commodity markets. Math. Finance, 21(4), pp 595-625.

[4] Benth, F. E., Šaltytè Benth, J., and Koekebakker, J. (2008). Stochastic Modelling of Electricity and Related Markets, World Scientific.

[5] Benth, F.E., Cartea, A., and Kiesel, R. (2008). Pricing forward contracts in power markets by the certainty equivalence principle: explaining the sign of the market risk premium. J. Banking Finance, 32(10), pp. 20062021.

[6] Benth, F. E., Di Nunno, G., and Khedher, A. (2010). Lévy model robustness and sensitivity. In $Q P-P Q$ : Quantum Probability and White Noise Analysis, Proceedings of the 29th Conference in Hammamet, Tunisia, 1318 October 2008, H. Ouerdiane and A Barhoumi (eds.), World Scientific, Vol. 25, pp. 153-184.

[7] Benth, F. E., Di Nunno, G., and Khedher, A. (2013). Computations of Greeks in multi-factor models with applications to power and commodity markets. J. Energy Markets, 5(4), pp. 3-31.

[8] Benth, F. E., Klüppelberg, C., Müller, G., and Vos, L. (2011). Futures pricing in electricity markets based on stable CARMA spot models. Submitted.

[9] Benth, F. E., and Koekebakker, S. (2008). Stochastic modeling of financial electricity contracts. Energy Economics, 30(3), pp. 1116-1157, 2008.

[10] Benth, F. E., Lempa, J., and Nilsen, T. K. (2012). On optimal exercise of swing options in electricity markets. J. Energy Markets, 4(4), pp. 3-28.

[11] Benth, F. E: and Meyer-Brandis, T. (2009). The information premium for non-storable commodities. J. Energy Markets, 2(3), pp. 111-140.

[12] Benth, F. E., and Sgarra, C. (2012). The risk premium and the Esscher transform in power markets. Stoch. Analysis Appl, 30, pp. 20-43.

[13] Brigo, D, and Mercurio, F. (2001). Interest Rate Models - Theory and Practice. Springer Verlag.

[14] Carmona, R., and Durrleman, V. (2003).Pricing and hedging spread options. SIAM Rev. 45, pp. $627-685$.

[15] Eydeland, A. and Wolyniec, K. (2003). Energy and Power Risk Management. John Wiley \& Sons.

[16] Folland, G. B. (1984). Real Analysis - Modern Techniques and their Applications. John Wiley \& Sons.

17] Geman, H. (2005). Commodities and Commodity Derivatives. Wiley-Finance.

[18] Heath, D., Jarrow, R., and Morton, A. (1992). Bond pricing and the term structure of interest rates: a new methodology. Econometrica, 60, 77-105.

[19] Hikspoors, S. and Jaimungal, S. (2008). Asymptotic pricing of commodity derivatives for stochastic volatility spot models. Appl. Math. Finance, 15(5\&6), pp. 449-467.

[20] Ikeda, N., and Watanabe, S. (1981). Stochastic Differential Equations and Diffusion Processes, NorthHolland/Kodansha.

[21] Karatzas, I., and Shreve, S. E. (1991). Brownian Motion and Stochastic Calculus, Springer Verlag.

[22] Lucia, J. and Schwartz, E. S. (2002). Electricity Prices and Power Derivatives: Evidence from the Nordic Power Exchange. Rev. Derivatives Res., 5(1), pp. 5-50.

[23] Margrabe, W. (1978). The value of an option to exchange one asset for another. J. Finance, 33, pp. 177-186.

[24] Nelsen, R. B. (2010). An Introduction to Copulas, Second Ed., Springer Verlag.

[25] Sato, K. (1999). Lévy Processes and Infinite Divisibility. Cambridge University Press.

[26] Schwartz, E. S. (1997). The stochastic behaviour of commodity prices: Implications for valuation and hedging. J. Finance, LII(3), pp. 923-973.

[27] Schwartz, E. S., and Smith, J. E. (2000). Short-term variations and long-term dynamics in commodity prices. Manag. Science, 46(7), pp. 893-911.

[28] Trolle, A. B., and Schwartz, E. S. (2009). Unspanned stochastic volatility and the pricing of commodity derivatives. Rev. Financial Studies, 22(11), pp. 4423-4461.

Center of Mathematics for Applications (CMA), University of Oslo, PO Box 1053 Blindern, N-0316

OSLO, NorWAY

E-mail address: fredb@math.uio.no

$U R L:$ http://folk.uio.no/fredb/ 